\title{
On a sparse random graph with minimum degree three: Likely Pósa sets are large
}

\author{
Alan Frieze* ANd Boris PitTel ${ }^{\dagger}$
}

\begin{abstract}
We consider the endpoint sets produced by Pósa rotations, when applied to a longest path in a random graph with $c n$ edges, conditioned on having minimum degree at least three. We prove that, for $c \geq 2.7$, the Pósa sets are likely to be almost linear in $n$, implying that the number of missing edges, each allowing either to get a longer path or to form a Hamilton cycle, is almost quadratic in $n$.

AMS 2000 SUBJECT ClASSificATIONS: 05C80, 05C30, 34E05, $60 \mathrm{C} 05$.

KEYWORDS AND PHRASES: Random, sparse graphs, degrees, longest path, Pósa sets.
\end{abstract}

\section{Introduction}

In the pioneering paper [10] Erdős and Rényi asked how large $m$, the number of edges, should be for the uniformly random graph on $n$ vertices $(G(n, m))$ with high probability (whp) to have a Hamilton cycle. The problem was vigorously attacked by the various authors, see references in Bollobás [6]; in particular, Komlós and Szemerédi [18] showed that $m=n^{1+\varepsilon}$ suffices. A critical breakthrough was achieved by Pósa [23]; he showed that $m=c n \ln n, c>30$, is enough. Qualitatively this is the best possible, since $m=\Theta(n \ln n)$ edges are needed for $G(n, m)$ to be connected whp, [10]. Progressively stronger extensions of Pósa's result for $G(n, m)$ were achieved by Korshunov [19], Komlós and Szemerédi [20], Ajtai, Komlós and Szemerédi [1], Bollobás [7], Bollobás, Fenner and Frieze [9], and Bollobás and Frieze [3]. The proofs frequently used a deceptively simple but surprisingly potent "Pósa's Lemma" from [23].

Here is the Lemma. Given a graph $G$ and a vertex $x_{0}$, let $P=x_{0} x_{1} \ldots x_{h}$ be a longest path from $x_{0}$. If $\left(x_{h}, x_{i}\right) \in E(G)$ for some $i<h-1$, then $P^{\prime}=x_{0} \ldots x_{i} x_{h} x_{h-1} \ldots x_{i+1}$ is also a path in $G$ from $x_{0}$, of the same edge length $h$. In words, $P^{\prime}$ is obtained from $P$ by rotation via the edge $\left(x_{i}, x_{h}\right)$.

*Frieze's research supported in part by NSF Grant CCF 2013110.

†Pittel's research supported in part by NSF Grants DMS 0805996, DMS 1101237. 
Let $S$ consist of $x_{h}$ and the set of endpoints of all paths obtainable from $P$ through any number of rotations. Let $T$ be the set of all outside neighbors of $S$ on the path $P$, "outside" meaning that $T \cap S=\emptyset$. Pósa's Lemma states that there are no edges between $S$ and $V(P) \backslash(S \cup T)$. Since by definition of $P, S$ has no neighbors outside of $P$, it follows from Pósa's Lemma that $T=N(S)$, the set of all outside neighbors of $S$. In addition,

$$
|T|<2|S|
$$

Now if a graph $G$ is not too sparse, one may expect that the not-too-large vertex sets $A$ are sufficiently expanding, so that $|N(A)| \geq 2|A|$. If that is the case, then it follows from Pósa's Lemma that $|S|$ has to exceed a threshold value dependent on $G, s=s(G)$, say. Bollobás's next crucial observation was that, if a path $P$ of length $h$ cannot be extended via a sequence of rotations at either of its ends then there are at least $\left(\begin{array}{c}s(G) \\ 2\end{array}\right)$ "non-edges" with a property: adding any such non-edge to $E(G)$ creates a cycle out of a properly rotated $P$. Connectivity considerations now lead to the conclusion that such a non-edge will either create a Hamilton cycle or enable us to find a path of length $h+1$. (The term "boosters" has recently been coined to describe these edges.) Using these fundamental properties of Pósa's sets, and also de la Vega's theorem on whp existence of long paths in $G(n, p),(n p>4 \ln 2),[12]$, Bollobás [7, 6] found a surprisingly direct proof of Korshunov's, Komlós-Szemerédi's result on a sharp threshold value of $m$ and $p$ for whp-Hamiltonicity of $G(n, m)$ and $G(n, p)$.

Specifically, he proved that, for $p$ in question, whp $s(G(n, p))=\Theta(n)$, that is the likely number of those beneficial non-edges is quadratic in $n$. Using this, he identified a sequence $G\left(n, p_{0}\right) \subset G\left(n, p_{1}\right) \subset G\left(n, p_{k}\right) \subset G(n, p)$, $k=k(n)$, such that de la Vega's result applies to $G\left(n, p_{0}\right)$ with room to spare, and for each $j$, with the conditional probability $1-O\left(n^{-2}\right)$, the length of the longest path in $G\left(n, p_{j+1}\right)$ strictly exceeds that in $G\left(n, p_{j}\right)$, if the latter is non-Hamiltonian. That $G\left(n, p_{k}\right)$ is whp Hamiltonian was then immediate.

Later we used a broadly similar argument to show that a random graph with minimum degree at least $2, G^{(2)}(n, m)$ in short, whp has a perfect matching iff $G^{(2)}(n, m)$ has no isolated odd cycles, see Frieze and Pittel [16]. A counterpart of Pósa's Lemma in our case was a lemma inspired by GallaiEdmonds Structure Theorem and Edmonds' Matching algorithm. This lemma allowed us to prove that, in absence of a perfect matching, with high conditional probability there exist $\Theta\left(n^{2}\right)$ non-edges each of which would increase the maximum matching number. And the place of de la Vega's algorithm was taken up by Karp-Sipser Matching Greedy [17], analyzed in detail in our earlier paper [2]. 
As its title indicates, the core of Bollobás' paper [7] was a proof that the uniformly random $d$-regular graph on $[n], G_{d}(n)$, is whp Hamiltonian, if $d>10^{7}$. Fenner and Frieze [11] independently proved that $d>796$ suffices and then Frieze [13] came up with an algorithmic proof of a better bound $d>85$. It was commonly believed that $d \geq 3$ suffices, and indeed Robinson and Wormald [24] settled this conjecture affirmatively. Their nonalgorithmic proof was based on a refined version of the second order moment prompted by their discovery that, for $d \geq 3, \mathrm{E}\left[X_{n}^{2}\right]=O\left(\mathrm{E}^{2}\left[X_{n}\right]\right), X_{n}$ being the number of Hamilton cycles. (The random graphs $G(n, p), G(n, m)$, with $p, m$ in question, lack this remarkable property.) Very recently, Bohman and Frieze [3] proved that another well-known random graph, $G_{d-\text { out }}(n)$ is whp Hamiltonian, if $d \geq 3$, in which case the average vertex degree is asymptotic to $2 d$.

Now, consider the Hamiltonicity property of $G^{(3)}(n, m)$, the random graph on $[n]$ with $m$ edges and minimum degree 3 , at least. Of course, $m \geq 3 n / 2$, and we had better assume $m>3 n / 2$, since equality implies that $G^{(3)}(n, m)=G_{3}(n)$. From a more general result in Bollobás, Cooper, Fenner and Frieze [8] it follows that whp $G^{(3)}(n, m)$ is Hamiltonian if $m>128 n$. Our ultimate goal is to push this bound down, close to the best possible $m>1.5 n$, and to construct an algorithm that finds a Hamilton cycle in $O_{p}\left(n^{1+o(1)}\right)$ running time for $m / n$ in the arising range.

Here is our main result in this paper.

For $k \geq 0$, let

$$
f_{k}(x)=\sum_{j \geq k} \frac{x^{j}}{j !}
$$

a tail of the series for $e^{x}$.

Theorem 1.1. Introduce $x^{*} \approx 4.789771 \ldots$, the unique positive root of

$$
\frac{x^{3} f_{1}(x)}{f_{2}(x)^{2}}=1,
$$

and

$$
a^{*}=\frac{x^{*} f_{2}\left(x^{*}\right)}{2 f_{3}\left(x^{*}\right)} \approx 2.6616 .
$$

If $m \geq\left(a^{*}+\varepsilon\right) n, \varepsilon>0$, i.e. the average vertex degree exceeds 5.32 , then whp for each pair of Pósa's sets $(S, T)$,

$$
|S|+|T| \geq n^{1-\delta_{n}}, \quad \delta_{n}=(\ln \ln n)^{-1 / 2} .
$$

In words, the likely Pósa's sets are, at least, almost linear in size. 
Remark 1.1. If the vertex degree range is an arbitrary $D \subseteq[3, \infty), 3 \in D$, then the above assertion continues to hold if we replace $f_{3}(x)$ with

$$
f_{D}(x)=\sum_{j \in D} \frac{x^{j}}{j !}
$$

and $f_{2}(x), f_{1}(x)$ with $f_{D}^{\prime}(x)$ and $f_{D}^{\prime \prime}(x)$ respectively. For instance, if $D=$ $\{3,4\}$, then the likely Pósa's sets are almost linear in size if $m / n \geq 17 / 9 \approx$ 1.9 .

Remark 1.2. Our calculations will reveal that the dominant contribution to a bound for the expected number of sparse Pósa sets $(S, T)$ comes from the pairs $(S, T)$ with a rather rigid structure of the subgraph induced by $S \cup T$; see Remark 4.1. Since this dominant contribution is bounded rather sharply, a further progress toward $m>3 n / 2$, or even $m / n>a, a \in\left(3 / 2, a^{*}\right)$, seems to be out of sight at this moment.

The proof of this claim takes up the rest of the paper. It is quite technical, apparently due to exceeding sparseness of $G^{(3)}(n, m)$ for $m / n$ that close to the best 1.5 . We firmly believe that, in a complete analogy with Bollobás' proof of Hamiltonicity of $G(n, p)$ and $G(n, m)$, and our result on the existence of a perfect matching in $G^{(2)}(n, m)$, the random graph $G^{(3)}(n, m)$ is whp Hamiltonian if $m \geq\left(a^{*}+\varepsilon\right) n$.

In the companion paper [14], instead of using extensions and rotations to grow a long path, we use them to convert a good 2-matching into a hamilton cycle. A 2-matching is a spanning subgraph of $G^{(3)}(n, m)$ with maximum degree at most two. By good, we mean that it has $O(\log n)$ components and the number of components is a good measure of how far it is away from being Hamiltonian. In this context, a path of length $\ell$ has $n-\ell+1$ components. To find the good 2-matching, we have found a modification of Karp-Sipser's Greedy Matching algorithm.

A simple way to use the results of this paper to prove Hamiltonicity goes like this. Hold back a random edge subset $X$ of cardinality $o\left(n^{1 / 2}\right)$, and then argue that the results of this paper apply to the remaining random graph. Then we know that whp there are always $\Omega\left(n^{2-o(1)}\right)$ boosters that can be used to move the extension-rotation algorithm along. The edges in $X$ are quite likely to be boosters (at least likely enough) and because our 2-matching is good, we only need a boost $O\left(\log ^{2} n\right)$ times (for details see [14]), and the mission can be completed.

One can go further though. Another paper, [15] shows how to use this 2-matching algorithm as a basis for finding a Hamilton cycle in $O\left(n^{1+o(1)}\right)$ time when $c$ is sufficiently large. 


\section{Pósa sets in a graph with minimum degree 3 at least}

Given a graph $G=(V, E)$ and $A \subseteq V$, we use $G(A)$ to denote the subgraph of $G$ induced by $A$, and $e(A)$ to denote the number of edges in $G(A)$.

Lemma 2.1. Suppose that the minimum degree of $G$ is 3 at least. Let $S, T$ be derived from a longest path $P$ as explained prior to Theorem 1.1. Then

$$
e(S \cup T)>|S \cup T|,
$$

i.e. the edge density of $G(S \cup T)$ strictly exceeds 1.

Proof of Lemma 2.1. Let $Q$ be any path obtained from $P$ by rotations. Pósa observed that

$$
\text { every } t \in T \text { has an } S \text {-neighbor on } Q \text {. }
$$

Introduce $T_{1}$, the set of all vertices $t \in T$ such that $t$ has only one neighbor $s \in S$. Pósa's observation implies that, for every such pair $(s, t), t$ and $s$ are neighbors on every path $Q$. In particular, when $s$ is an endpoint, $t$ is next to $s$. It follows then that for any other vertex $t^{\prime} \in T_{1}$ with a single neighbor $s^{\prime} \in S$ we have $s^{\prime} \neq s$. Therefore $\left|T_{1}\right| \leq|S|$.

Let $D(S)$ denote the total degree of vertices in $S$, and let $D_{S}(T)(\leq$ $D(S)$ ) denote the total number of edges with one endpoint in $T$ and another endpoint in $S$. Since each $t \in T \backslash T_{1}$ has at least two neighbors in $S$, and $\left|T_{1}\right| \leq|S|$, we have

$$
D_{S}(T) \geq\left|T_{1}\right|+2\left(|T|-\left|T_{1}\right|\right) \geq 2|T|-|S| .
$$

Hence, as each $s \in S$ has degree 3 at least,

$$
D(S)+D_{S}(T) \geq 3|S|+2|T|-|S|=2(|S|+|T|) .
$$

As

$$
2 e(S \cup T)=D(S)+D_{S}(T)+2 e(T),
$$

$e(T)$ being the number of edges in the subgraph $G(T)$ induced by $T$, we see that

$$
e(S \cup T) \geq|S \cup T| .
$$

The rest of the argument is needed to upgrade this to the strict inequality. 
From the proof of (2.4), and (2.5), it follows that the edge density of $G(S \cup T)$ may be equal 1 only if

(a)

$$
D(S)+D_{S}(T)=2(|S|+|T|)
$$

(b) there are no edges in $T$;

(c) $\left|T_{1}\right|=|S|$;

(d) each vertex in $S$ has exactly two neighbors in $S \cup\left(T \backslash T_{1}\right)$;

(e) each vertex in $\widehat{T}_{1}:=T \backslash T_{1}$ has exactly two neighbors in $S$.

If one of (c), (d), (e) is violated then $D(S)+D_{S}(T)>2(|S|+|T|)$.

Case $\widehat{T}_{1}=\emptyset$. Given a path $P$, the $S$-vertices are distributed over $P$ as subpaths of vertex length $i \geq 1$, next neighbors of subpaths being $T_{1}$-vertices. Since $T_{1}$-vertices remain the neighbors of their single $S$-neighbors on every path, and $\left|T_{1}\right|=|S|$, there can be only paths of length 1 and 2 , "monomers" and "dimers". An endpoint $s$ of $P$ is a monomer, as its left neighbor is $t \in T_{1}$. There are no other monomers in $P$, since an interior monomer would be flanked by two $T_{1}$-vertices, sharing a common neighbor in $S$, which is impossible. Consequently, the leftmost subpath of $P$ is a dimer $s_{1}, s_{2}$, sandwiched between two vertices $t_{1}, t_{2} \in T_{1}$. No rotation from $P$ can use either $t_{i}$, as $s_{i}$ is the only $S$-neighbor of $t_{i}$, or $s_{2}$, as $t_{2} \notin S$. If the rotation uses $s_{1}$, then $t_{1}, s_{1}, s, t$ becomes the new leftmost dimer with $s_{1}$ retaining the left position. Of course, if a rotation does not use $s_{1}$, then $t_{1}, s_{1}, s_{2}, t_{2}$ remains the leftmost subpath. So no sequence of rotations will make $s_{1}$ an endpoint. Contradiction.

Case $\widehat{T}_{1} \neq \emptyset$. By $(\mathrm{d})-(\mathrm{e})$, the graph $G\left(S \cup \widehat{T}_{1}\right)$ is a disjoint union of cycles. By (b), each cycle contains at least two vertices from $S$. In fact, there is just one cycle, since otherwise there would exist two vertices $s_{1}, s_{2} \in S$ such that no sequence of rotations starting with a path with the endpoint $s_{1}$ would lead to a path ending at $s_{2}$.

Since there are no edges between vertices in $\widehat{T}_{1}$, two vertices from $\widehat{T}_{1}$ cannot be neighbors on the cycle. And no two vertices from $S$ can be neighbors either. Otherwise, there is an arc $s_{1} s_{2} t$, with $s_{1}, s_{2} \in S, t \in \widehat{T}_{1}$. Consider a path $Q$ that ends at $s_{1}$. We know that the left neighbor of $s_{1}$ in $Q$ is a $t_{1} \in T_{1}$. By considering the rotation from $Q$ via the edge $\left(s_{1}, s_{2}\right)$ we see that $s_{2}$ has another neighbor $s_{3} \in S$ distinct from $s_{1}$. Hence $s_{2}$ has at least three neighbors in $S \cup \widehat{T}_{1}$, namely $s_{1}, s_{3}, t$. This violates (d). 
Therefore the vertices from $S$ and from $\widehat{T}_{1}$ alternate on the cycle. Hence $\left|\widehat{T}_{1}\right|=|S|$, whence

$$
|T|=\left|T_{1}\right|+\left|\widehat{T}_{1}\right|=2|S|,
$$

which violates Pósa inequality (1.1).

So the edge density of $G(S \cup T)$ exceeds 1 .

Remark 2.1. The above argument needs to be refined if we want to put a bound on the time taken to construct the end-point sets. In this case suppose that we are doing a sequence of rotations with fixed endpoint $v_{0}$. If a rotation would produce an endpoint that has been produced before in this sequence, then we do not do this rotation. This limits the time spent producing endpoints, but it will reduce the number of endpoints, but we will now argue that Lemma 2.1 continues to hold. Indeed, all we have to observe is that (2.2) continues to hold. The argument being identical to Posá's argument.

In the course of the proof, having assumed that the edge density of $G(S \cup T)$ is 1 , we saw that then $G(S \cup T)$ must be quite special. Namely $\left|T_{1}\right|=|S|$, and either $(1) \widehat{T}_{1}=\emptyset$ and $G(S \cup T)$ is a cycle on $S$, with each of $T_{1}$-vertices attached to its own $S$-vertex, or $(2)\left|\widehat{T}_{1}\right|=|S|$, and $G(S \cup T)$ is an alternating cycle on a bipartition $\left(S, \widehat{T}_{1}\right)$, with each of $S$-vertices hosting its own pendant vertex from $T_{1}$. The punch line was that neither of these two graphs, each of edge density 1, can be a Pósa graph $G(S \cup T)$.

In the next section we will show that in the random graph $G^{(3)}(n, m)$ whp no vertex subset $A$, with $|A| \leq \varepsilon_{0} \ln n$ can induce a subgraph of edge density exceeding 1. So, by (1.1) and Lemma 2.1, whp $|S|+|T|>\varepsilon_{0} \ln n$. We will also show that whp the edge density of the induced subgraph is $1+o(1)$, for every $A$, with $\varepsilon_{0} \ln n<|A| \leq n^{1-o(1)}$. It is natural then to focus on the $o(n)$ Pósa sets of edge density close to 1 , anticipating that the induced subgraphs $G(S \cup T)$ should interpolate between those two special, impossible, graphs. To prepare, let us have a look at the deterministic properties of $G(S \cup T)$ with an edge density close to 1 .

Introduce $G^{*}=G^{*}\left(S \uplus\left(T \backslash T_{1}\right)\right)$, the subgraph on the vertex set $S \cup$ $\left(T \backslash T_{1}\right)$ whose edges have at least one end in $S$; so we disregard edges of $G(S \cup T)$ between vertices of $T$, and also edges joining the pendant vertices of $T_{1}$ to their respective $S$ - "hosts". For $v \in S \cup\left(T \backslash T_{1}\right)$, let $d\left(v ; G^{*}\right)$ denote the degree of $v$ in $G^{*}$; by the definition of $G^{*}, \min _{v} d\left(v ; G^{*}\right) \geq 2$. Introduce

$$
S_{2}=\left\{v \in S: d\left(v ; G^{*}\right)=2\right\} .
$$




\section{Lemma 2.2.}

(i) No vertex from $S_{2}$ can be a neighbor of both a vertex in $S_{2}$ and a vertex in $T \backslash T_{1}$.

(ii) Suppose that

$$
e(S \cup T)=(1+\sigma)(s+t), \quad s:=|S|, t:=|T|,
$$

for some $\sigma>0$. Then, denoting $\left|T_{1}\right|=t_{1}$,

$$
\begin{aligned}
s-2 \sigma(s+t) & \leq t_{1} \leq s, \\
\sum_{v \in S \cup\left(T \backslash T_{1}\right)}\left[d\left(v ; G^{*}\right)-2\right] & \leq 2 \sigma(s+t) .
\end{aligned}
$$

Remark. Recalling that $t<2 s$, the bound (2.7) is not vacuous if $\sigma \leq 1 / 6$. Proof of Lemma 2.2. (i) Suppose that there are $s_{1}, s_{2} \in S_{2}$ and $t \in T \backslash T_{1}$ such that $\left(s_{1}, s_{2}\right)$ and $\left(s_{1}, t\right)$ are edges in $G^{*} . s_{2}$ has a neighbor $t_{1} \in T_{1}$, since $s_{2}$ 's degree in $G(S \cup T)$ is at least, whence exactly, 3. Consider a path $P$ with $s_{2}$ as its endpoint. $t_{1}$ is necessarily a penultimate vertex of $P$. Rotating $P$ via the edge $\left(s_{1}, s_{2}\right)$ must make the right $P$-neighbor of $s_{1}$ a new endpoint. (Here we assume that $x_{i+1}$ is to the right of $x_{i}$ for $i \geq 0$ ). So $s_{1}$ has a neighbor in $S$ distinct from $s_{2}$, and $d\left(s_{1} ; G^{*}\right) \geq 3$. Contradiction.

(ii) First, using (2.3), $D(S) \geq 3 s$, and (2.5), we obtain

$$
2(s+t)+\left(s-t_{1}\right) \leq 2 e(S \cup T)=2(1+\sigma)(s+t)
$$

which implies $(2.7)$ as $s-t_{1} \geq 0$. Second, the total vertex degree of $G^{*}$ is

$$
\sum_{v \in S \cup\left(T \backslash T_{1}\right)} d\left(v ; G^{*}\right)=2 e(S \cup T)-2 t_{1}-2 e(T) .
$$

Therefore

$$
\begin{aligned}
\sum_{v \in S \cup\left(T \backslash T_{1}\right)}\left[d\left(v ; G^{*}\right)-2\right] & =2 e(S \cup T)-2 t_{1}-2 e(T)-2\left(s+t-t_{1}\right) \\
& =2 e(S \cup T)-2(s+t)-2 e(T) \\
& =2(1+\sigma)(s+t)-2(s+t),
\end{aligned}
$$

which implies (2.8). 
Introduce

$$
\begin{aligned}
& S_{3}:=S \backslash S_{2}, \\
& T_{2}:=\left\{v \in T \backslash T_{1}: d\left(v ; G^{*}\right)=2\right\}, \quad T_{3}:=\left(T \backslash T_{1}\right) \backslash T_{2},
\end{aligned}
$$

and denote $s_{i}=\left|S_{i}\right|, t_{i}=\left|T_{i}\right|$; so $s=s_{2}+s_{3}, t=t_{1}+t_{2}+t_{3}$. It follows from (2.8) that

$$
\sum_{v \in S_{3} \cup T_{3}}\left[d\left(v ; G^{*}\right)-2\right] \leq 2 \sigma(s+t)
$$

and then

$$
\left|S_{3} \cup T_{3}\right|=s_{3}+t_{3} \leq 2 \sigma(s+t)
$$

Let $\mu_{1}$ denote the total number of edges in the subgraph of $G^{*}(S \uplus(T)$ $\left.T_{1}\right)$ ) induced by $S$, and let $\mu_{2}$ denote the total number of the remaining edges of $G^{*}\left(S \uplus\left(T \backslash T_{1}\right)\right)$, those joining vertices of $S$ and $T \backslash T_{1}$, and set $\mu=\mu_{1}+\mu_{2}$. Clearly

$$
\begin{aligned}
2 \mu_{1}+\mu_{2} & =\sum_{v \in S} d\left(v ; G^{*}\right), \\
\mu_{2} & =\sum_{v \in T \backslash T_{1}} d\left(v ; G^{*}\right) .
\end{aligned}
$$

Adding the equations (2.12) and (2.13),

$$
\begin{aligned}
\mu & :=\frac{1}{2} \sum_{v \in(S \cup T) \backslash T_{1}} d\left(v ; G^{*}\right)=s_{2}+t_{2}+\frac{1}{2} \sum_{v \in S_{3} \cup T_{3}} d\left(v ; G^{*}\right) \\
& =s+t-t_{1}+\frac{\xi_{1}+\xi_{2}}{2}
\end{aligned}
$$

where

$$
\xi_{1}:=\sum_{v \in S_{3}}\left[d\left(v ; G^{*}\right)-2\right] \geq s_{3}, \quad \xi_{2}:=\sum_{v \in T_{3}}\left[d\left(v ; G^{*}\right)-2\right] \geq t_{3}
$$

It follows from $(2.12),(2.13)$ and $(2.15)$ that

$$
\begin{aligned}
& \mu_{1}=s-t+t_{1}+\frac{\xi_{1}-\xi_{2}}{2}, \\
& \mu_{2}=2\left(t-t_{1}\right)+\xi_{2} .
\end{aligned}
$$


From (2.10),

$$
\xi_{1}+\xi_{2} \leq 2 \sigma(s+t)
$$

Remark 2.2. We note here that Lemma 2.2 continues to hold under the restrictions described in Remark 2.1.

Let two disjoint sets, $S$ and $T$, the partitions $S=S_{2} \cup S_{3}, T=T_{1} \cup T_{2} \cup T_{3}$, and $\xi_{1}, \xi_{2}$ be given. Let $\mathcal{N}(\mathbf{S}, \mathbf{T}, \boldsymbol{\xi})$ denote the total number of the subgraphs $G^{*}\left(S \uplus\left(T \backslash T_{1}\right)\right)$, with $\mu_{1}, \mu_{2}$ determined by (2.16), such that the constraints (2.7), whence the constraints (2.10), (2.11) and (2.17) hold for some $\sigma>0$.

\section{Lemma 2.3.}

(i)

$$
\begin{aligned}
& \mathcal{N}(\mathbf{S}, \mathbf{T}, \boldsymbol{\xi}) \leq \mathcal{N}_{1}(\mathbf{s}, \mathbf{t}, \boldsymbol{\xi}) \\
& \mathcal{N}_{1}(\mathbf{s}, \mathbf{t}, \boldsymbol{\xi}):=2^{-s_{2}-t_{2}-\mu_{1}} \frac{\left(2 \mu_{1}+\mu_{2}\right) !}{\mu_{1} !} \exp [O(\sigma(s+t))] .
\end{aligned}
$$

(ii) There exists $\sigma_{0} \in(0,1)$ such that, for $\sigma \leq \sigma_{0}$ and

$$
\left(1+\sigma^{1 / 2}\right) s \leq t \leq 2\left(1-\sigma^{1 / 2}\right) s,
$$

a stronger bound holds:

$$
\begin{aligned}
& \mathcal{N}(\mathbf{S}, \mathbf{T}, \boldsymbol{\xi}) \leq \mathcal{N}_{2}(\mathbf{s}, \mathbf{t}, \boldsymbol{\xi}):=\mathcal{N}_{1}(\mathbf{s}, \mathbf{t}, \boldsymbol{\xi})(s+t)^{2} \\
\times & \exp \left[-(2 s-t) \ln \frac{s}{2 s-t}-(t-s) \ln \frac{s}{t-s}+O\left(\sigma^{1 / 2}(s+t)\right)\right] .
\end{aligned}
$$

Proof of Lemma 2.3. It is well known, see Bollobás [4], that $g(\mathbf{d})$, the total number of graphs on $[\nu]$ with vertex degrees $d_{1}, \ldots, d_{\nu}$, and total vertex degree $2 M:=\sum_{i} d_{i}$ satisfies

$$
g(\mathbf{d}) \leq(2 M-1) ! ! \prod_{i=1}^{\nu} \frac{1}{d_{i} !} .
$$

Here is a bipartite counterpart of $(2.21)$. Let $\nu_{1}, \nu_{2}$, and $\mathbf{d}^{\prime}=\left(d_{1}^{\prime}, \ldots, d_{\nu_{1}}^{\prime}\right)$, $\mathbf{d}^{\prime \prime}=\left(d_{1}^{\prime \prime}, \ldots, d_{\nu_{2}}^{\prime \prime}\right)$ be such that

$$
\sum_{i \in\left[\nu_{1}\right]} d_{i}^{\prime}=\sum_{j \in\left[\nu_{2}\right]} d_{j}^{\prime \prime}=M
$$


Denote by $g\left(\mathbf{d}^{\prime}, \mathbf{d}^{\prime \prime}\right)$ the total number of bipartite graphs on a bipartition $\left[\nu_{1}\right] \uplus\left[\nu_{2}\right]$, with the left vertices and the right vertices having degrees $\mathbf{d}^{\prime}$ and $\mathbf{d}^{\prime \prime}$. Then

$$
g\left(\mathbf{d}^{\prime}, \mathbf{d}^{\prime \prime}\right) \leq M ! \prod_{i \in\left[\nu_{1}\right]} \frac{1}{d_{i}^{\prime} !} \prod_{j \in\left[\nu_{2}\right]} \frac{1}{d_{j}^{\prime \prime !}}
$$

(i) Let $\mathbf{d}=\left\{d_{v}\right\}_{v \in S}$ be the (generic) vertex degrees of the subgraph of $G^{*}\left(S \uplus\left(T \backslash T_{1}\right)\right)$ induced by $S$; so

$$
\sum_{v \in S} d_{v}=2 \mu_{1}
$$

Let $\mathbf{d}^{\prime}=\left\{d_{v}^{\prime}\right\}_{v \in S}$ and $\mathbf{d}^{\prime \prime}=\left\{d_{v}^{\prime \prime}\right\}_{v \in T_{2} \cup T_{3}}$ denote the vertex degrees of the complementary bipartite graph on the bipartition $S \uplus\left(T_{2} \cup T_{3}\right)$; so

$$
\sum_{v \in S} d_{v}^{\prime}=\sum_{v \in T_{2} \cup T_{3}} d_{v}^{\prime \prime}=\mu_{2}
$$

Here $\mu_{1}, \mu_{2}, \mu=\mu_{1}+\mu_{2}$ are given by (2.12), (2.13) and (2.14). In addition,

$$
d_{v}+d_{v}^{\prime} \begin{cases}=2, & v \in S_{2} \\ \geq 3, & v \in S_{3}\end{cases}
$$

and

$$
d_{v} \begin{cases}=2, & v \in T_{2} \\ \geq 3, & v \in T_{3} .\end{cases}
$$

Using (2.21) and (2.22), we get an upper bound for the number of the graphs with vertex degrees $\mathbf{d}, \mathbf{d}^{\prime}, \mathbf{d}^{\prime \prime}$ :

$$
\left(2 \mu_{1}-1\right) ! ! \mu_{2} ! \prod_{v \in S} \frac{1}{d_{v} ! d_{v}^{\prime} !} \prod_{v \in T_{2} \cup T_{3}} \frac{1}{d_{v}^{\prime \prime !}}
$$

Introducing

$$
f_{k}(x)=\sum_{j \geq k} \frac{x^{j}}{j !}
$$


we have then

$$
\begin{gathered}
\sum_{\substack{\mathbf{d}, \mathbf{d}^{\prime}, \mathbf{d}^{\prime \prime} \text { meet } \\
(2.23)-(2.26)}} \prod_{v \in S} \frac{1}{d_{v} ! d_{v}^{\prime} !} \prod_{v \in T_{2} \cup T_{3}} \frac{1}{d_{v}^{\prime \prime !}} \\
=2^{-t_{2}}\left[x^{2 \mu_{1}} y^{\mu_{2}}\right]\left(\sum_{d+d^{\prime}=2} \frac{x^{d} y^{d^{\prime}}}{d ! d^{\prime} !}\right)^{s_{2}}\left(\sum_{d+d^{\prime} \geq 3} \frac{x^{d} y^{d^{\prime}}}{d ! d^{\prime} !}\right)^{s_{3}} \cdot\left[z^{\mu_{2}-2 t_{2}}\right]\left(\sum_{d^{\prime \prime} \geq 3} \frac{z^{d^{\prime \prime}}}{d^{\prime \prime !} !}\right)^{t_{3}} \\
=2^{-t_{2}}\left[x^{2 \mu_{1}} y^{\mu_{2}}\right]\left[\frac{(x+y)^{2}}{2}\right]^{s_{2}}\left[f_{3}(x+y)\right]^{s_{3}} \cdot\left[z^{\mu_{2}-2 t_{2}}\right]\left[f_{3}(z)\right]^{t_{3}} \\
=2^{-s_{2}-t_{2}}\left(\begin{array}{c}
2 \mu_{1}+\mu_{2} \\
\mu_{2}
\end{array}\right)\left[\xi^{2 \mu_{1}+\mu_{2}}\right] \xi^{2 s_{2}} f_{3}(\xi)^{s_{3}} \cdot\left[z^{\mu_{2}-2 t_{2}}\right]\left[f_{3}(z)\right]^{t_{3}} \\
=2^{-s_{2}-t_{2}}\left(\begin{array}{c}
2 \mu_{1}+\mu_{2} \\
\mu_{2}
\end{array}\right)\left[\xi^{2 \mu_{1}+\mu_{2}-2 s_{2}}\right] f_{3}(\xi)^{s_{3}} \cdot\left[z^{\mu_{2}-2 t_{2}}\right]\left[f_{3}(z)\right]^{t_{3}} \\
\leq 2^{-s_{2}-t_{2}}\left(\begin{array}{c}
2 \mu_{1}+\mu_{2} \\
\mu_{2}
\end{array}\right) f_{3}(1)^{s_{3}+t_{3}} .
\end{gathered}
$$

Thus

$$
\begin{aligned}
\mathcal{N}(\mathbf{S}, \mathbf{T}, \boldsymbol{\xi}) \leq\left(2 \mu_{1}-1\right) ! ! \mu_{2} ! 2^{-s_{2}-t_{2}}\left(\begin{array}{c}
2 \mu_{1}+\mu_{2} \\
\mu_{2}
\end{array}\right) f_{3}(1)^{s_{3}+t_{3}} & \\
& =2^{-s_{2}-t_{2}} \frac{\left(2 \mu_{1}-1\right) ! !}{\left(2 \mu_{1}\right) !}\left(2 \mu_{1}+\mu_{2}\right) ! f_{3}(1)^{s_{3}+t_{3}}
\end{aligned}
$$

So, by $(2.15)$ and $(2.17)$, and using $\left(2 \mu_{1}-1\right) ! !=\left(2 \mu_{1}\right) ! / 2^{\mu_{1}} \mu_{1} !$,

$(2.27) \mathcal{N}(\mathbf{S}, \mathbf{T}, \boldsymbol{\xi}) \leq \mathcal{N}_{1}(\mathbf{s}, \mathbf{t}, \boldsymbol{\xi}):=2^{-s_{2}-t_{2}-\mu_{1}} \frac{\left(2 \mu_{1}+\mu_{2}\right) !}{\mu_{1} !} \exp [O(\sigma(s+t))]$

(ii) Let $\left\{d_{v}\right\}_{v \in S_{2}},\left\{d_{v}\right\}_{v \in S_{3}}$ be the vertex degrees of the subgraphs of $G^{*}\left(S \uplus\left(T \backslash T_{1}\right)\right)$ induced by $S_{2}$ and $S_{3}$ respectively. Let $\left\{\delta_{v}\right\}_{v \in S_{2}},\left\{\delta_{v}\right\}_{v \in S_{3}}$ denote the vertex degrees of a bipartite graph induced by the bipartition $S_{2} \uplus$ $S_{3}$. Finally, let $\left\{d_{v}^{\prime}\right\}_{v \in S},\left\{d_{v}^{\prime \prime}\right\}_{v \in T \backslash T_{1}}$ denote the vertex degrees of a bipartite graph induced by the bipartition $S \uplus\left(T \backslash T_{1}\right)$. By the definition,

$$
\begin{aligned}
& d_{v}+\delta_{v}+d_{v}^{\prime}=2, \quad\left(v \in S_{2}\right), \quad d_{v}+\delta_{v}+d_{v}^{\prime} \geq 3, \quad\left(v \in S_{3}\right), \\
& d_{v}^{\prime \prime}=2, \quad\left(v \in T_{2}\right), \quad d_{v}^{\prime \prime} \geq 3, \quad\left(v \in T_{3}\right),
\end{aligned}
$$

and by Lemma 2.2

$$
d_{v} \cdot d_{v}^{\prime}=0, \quad v \in S_{2}
$$


Denote

$$
\begin{array}{lll}
\sum_{v \in S_{2}} d_{v}=2 \nu_{2}, & \sum_{v \in S_{2}} \delta_{v}=\nu_{2,3}, & \sum_{v \in S_{2}} d_{v}^{\prime}=\mu_{2,2} \\
\sum_{v \in S_{3}} d_{v}=2 \nu_{3}, & \sum_{v \in S_{3}} \delta_{v}=\nu_{3,2}, & \sum_{v \in S_{3}} d_{v}^{\prime}=\mu_{3,2}
\end{array}
$$

then $\nu_{2,3}=\nu_{3,2}$, and

$$
\begin{aligned}
& 2 \nu_{2}+\nu_{2,3}+\mu_{2,2}=2\left|S_{2}\right|=2 s_{2}, \\
& 2 \nu_{3}+\nu_{3,2}+\mu_{3,2}=2 \mu_{1}+\mu_{2}-2 s_{2}, \\
& \mu_{2,2}+\mu_{3,2}=\mu_{2}, \\
& \nu_{2}+\nu_{2,3}+\nu_{3}=\mu_{1} .
\end{aligned}
$$

Given the values of $\nu_{2}, \nu_{3}, \nu_{2,3}$, and $\mu_{2,2}, \mu_{3,2}$, the number of the corresponding subgraphs $G^{*}\left(S \uplus\left(T \backslash T_{1}\right)\right)$ is bounded, as in part (i), by

$$
\left(2 \nu_{2}-1\right) ! !\left(2 \nu_{3}-1\right) ! ! \nu_{2,3} ! \mu_{2} ! 2^{-t_{2}} f_{3}(1)^{t_{3}}
$$

$$
\times\left[x_{1}^{2 \nu_{2}} x_{2}^{\mu_{2,2}} x_{3}^{\nu_{2,3}}\right]\left(\sum_{\substack{d+d^{\prime}+\delta=2 \\ d \cdot d^{\prime}=0}} \frac{x_{1}^{d} x_{2}^{d^{\prime}} x_{3}^{\delta}}{d ! d^{\prime} ! \delta !}\right)^{s_{2}}
$$

$$
\times\left[y_{1}^{2 \nu_{3}} y_{2}^{\mu_{3,2}} y_{3}^{\nu_{3,2}}\right]\left(\sum_{d+d^{\prime}+\delta \geq 3} \frac{y_{1}^{d} y_{2}^{d^{\prime}} y_{3}^{\delta}}{d ! d^{\prime} ! \delta !}\right)^{s_{3}} .
$$

The last line factor is

$$
\begin{aligned}
\left(\begin{array}{c}
2 \nu_{3}+\mu_{3,2}+\nu_{3,2} \\
2 \nu_{3}, \mu_{3,2}, \nu_{3,2}
\end{array}\right) & {\left[y^{2 \nu_{3}+\mu_{3,2}+\nu_{3,2}}\right] f_{3}(y)^{s_{3}} } \\
\leq 3^{2 \nu_{3}+\mu_{3,2}+\nu_{3,2}} & f_{3}(1)^{s_{3}}=3^{2 \mu_{1}+\mu_{2}-2 s_{2}} f_{3}(1)^{s_{3}}
\end{aligned}
$$

By (2.16), this is

$$
2 \mu_{1}+\mu_{2}-2 s_{2}=2 s_{3}+\xi_{1}
$$

Now $s_{3} \leq \xi_{1}$, see (2.15), and so by (2.17) the RHS of (2.30) is bounded by

$$
3^{2 s_{3}+\xi_{1}} f_{3}(1)^{s_{3}} \leq\left(27 f_{3}(1)\right)^{\xi_{1}} \leq\left(27 f_{3}(1)\right)^{2 \sigma(s+t)} .
$$


The second line factor in (2.29) is bounded by

$$
\frac{\left(\frac{x_{1}^{2}}{2}+x_{1} x_{3}+\frac{\left(x_{2}+x_{3}\right)^{2}}{2}\right)^{s_{2}}}{x_{1}^{2 \nu_{2}} x_{2}^{\mu_{2,2}} x_{3}^{\nu_{2,3}}},
$$

for all $x_{1}, x_{2}, x_{3}>0$. The challenge is to select the "best" $x_{1}, x_{2}, x_{3}$. First of all $\mu_{3,2}, \nu_{2,3} \leq 3 \xi_{1}$, since $\nu_{2,3}=\nu_{3,2}$ and by (2.15), (2.28) and (2.31)

$$
2 \nu_{3}+\mu_{3,2}+\nu_{3,2} \leq 3 \xi_{1}
$$

Therefore, by (2.7), (2.10), (2.16), (2.17) and (2.28),

$$
\begin{aligned}
\nu_{2} & =\mu_{1}-\nu_{2,3}-\nu_{3} \leq 2 s-t+O(\sigma(s+t)), \\
\mu_{2,2} & =\mu_{2}-\mu_{3,2}=2(t-s)+O(\sigma(t+s)) .
\end{aligned}
$$

By the condition in the Lemma, the explicit terms are of order $\sigma^{1 / 2}(s+t)$ at least, thus dwarf the remainders if $\sigma$ is small. We pick

$$
x_{1}=\left(2 \nu_{2}\right)^{1 / 2}, \quad x_{2}=\left(\mu_{2,2}\right)^{1 / 2}, \quad x_{3}=\left(\nu_{2,3}\right)^{1 / 2} .
$$

Then

$$
\begin{aligned}
\frac{x_{1}^{2}}{2}+x_{1} x_{3}+\frac{\left(x_{2}+x_{3}\right)^{2}}{2} & =\frac{1}{2}\left(x_{1}^{2}+x_{2}^{2}+x_{3}^{2}\right)+\left(x_{1}+x_{2}\right) x_{3} \\
& =s_{2}+O(\sqrt{(s+t) \xi}) \\
& =s+O\left(\sigma^{1 / 2}(s+t)\right),
\end{aligned}
$$

as $s_{2}=s+O(\sigma(s+t))$. So the fraction in (2.33) can be bounded from above by

$$
\begin{aligned}
& \frac{1}{2^{s_{2}}} \sqrt{\frac{\left(2 s_{2}\right)^{2 s_{2}}}{\left(2 \nu_{2}\right)^{2 \nu_{2}} \mu_{2,2}^{\mu_{2,2}} \nu_{2,3}^{\nu_{2,3}}}} \exp \left(O\left(\sigma^{1 / 2}(s+t)\right)\right) \\
& =\frac{1}{2^{s_{2}}} \exp \left(\nu_{2} \ln \frac{s_{2}}{\nu_{2}}+\frac{\mu_{2,2}}{2} \ln \frac{s_{2}}{\mu_{2,2} / 2}+\frac{\nu_{2,3}}{2} \ln \frac{s_{2}}{\nu_{2,3} / 2}\right) \exp \left(O\left(\sigma^{1 / 2}(s+t)\right)\right) \\
& =\frac{1}{2^{s_{2}}} \exp \left[(2 s-t) \ln \frac{s}{2 s-t}+(t-s) \ln \frac{s}{t-s}+O\left(\sigma^{1 / 2}(s+t)\right)\right] .
\end{aligned}
$$


Turn to the first line of (2.29). Using $(2 a-1) ! ! \leq 2^{a} a$ !, and (2.28), (2.34), we get

$$
\begin{aligned}
\left(2 \nu_{2}-1\right) ! !\left(2 \nu_{3}-1\right) ! ! \nu_{2,3} ! \mu_{2} ! & \leq 2^{\nu_{2}+\nu_{3}} \nu_{2} ! \nu_{2,3} ! \nu_{3} ! \mu_{2} ! \leq 2^{\nu_{2}+\nu_{3}} \mu_{1} ! \mu_{2} ! \\
& =2^{\mu_{1}} \mu_{1} ! \mu_{2} ! \exp (O(\sigma(s+t))) .
\end{aligned}
$$

Putting together (2.29), (2.32), (2.36) and (2.37), we conclude that the number of subgraphs $G^{*}\left(S \uplus\left(T \backslash T_{1}\right)\right)$ with parameters $\boldsymbol{\mu}, \boldsymbol{\nu}$ is bounded by

$$
\frac{\mu_{1} ! \mu_{2} !}{2^{s_{2}+t_{2}-\mu_{1}}} \exp \left[H(s, t)+O\left(\sigma^{1 / 2}(s+t)\right)\right],
$$

where

$$
H(s, t)=(2 s-t) \ln \frac{s}{2 s-t}+(t-s) \ln \frac{s}{t-s} .
$$

We emphasize that the remainder term estimate is uniform over the range of the parameters $\boldsymbol{\xi}, \boldsymbol{\nu}$.

Let us compare the bounds (2.27) and (2.38). We have

$$
\frac{2^{-s_{2}-t_{2}-\mu_{1}} \frac{\left(2 \mu_{1}+\mu_{2}\right) !}{\mu_{1} !}}{2^{-s_{2}-t_{2}+\mu_{1}} \mu_{1} ! \mu_{2} !}=2^{-2 \mu_{1}}\left(\begin{array}{c}
2 \mu_{1}+\mu_{2} \\
\mu_{1}, \mu_{1}, \mu_{2}
\end{array}\right) .
$$

Using

$$
\mu_{1}=2 s-t+O(\sigma(s+t)), \quad \mu_{2}=2(t-s)+O(\sigma(s+t)),
$$

(cf. (2.34), (2.35)), and the Lemma condition on $2 s-t, t-s$, it is simple to show that the last expession is

$$
\exp \left[2 H(s, t)+O\left(\sigma^{1 / 2}(s+t)\right)\right] .
$$

Thus the bound (2.38) can be written as

$$
\mathcal{N}_{1}(\mathbf{s}, \mathbf{t}) \exp \left[-2 H(s, t)+O\left(\sigma^{1 / 2}(s+t)\right)\right] .
$$

The bound (2.40) implies (2.20), since the factor $(s+t)^{2}$ is an upper bound for the number of solutions $\left(\nu_{2}, \nu_{2,3}, \nu_{3}, \mu_{2,2}, \mu_{3,2}\right)$ of $(2.28)$.

Let $G^{* *}(S \cup T)$ denote $G^{*}\left(S \cup\left(T \backslash T_{1}\right)\right)$ adorned with $t_{1}$ pendant $T_{1}$ vertices attached to some $t_{1} S$-vertices. That is, $G^{* *}(S \cup T)$ is $G(S \cup T)$ without the edges joining $T$-vertices to each other. 
Given two disjoint sets $S$ and $T$, let $N(\mathbf{s}, \mathbf{t}, \boldsymbol{\xi})$ denote the total number of graphs $G^{* *}(S \cup T)$ with $\left|S_{2}\right|=s_{2},\left|T_{1}\right|=t_{1},\left|T_{2}\right|=t_{2}$ and the parameters $\xi_{1}, \xi_{2}$. The number of ways to select $T_{1} \subset T$ of cardinality $t_{1}$ and then to match the vertices of $T_{1}$ with some $t_{1}$ vertices in $S$ is $t_{1} !\left(\begin{array}{c}s \\ t_{1}\end{array}\right)\left(\begin{array}{c}t \\ t_{1}\end{array}\right)$. The number of ways to select $S_{2} \subset S$ of cardinality $s_{2}$ and to select $T_{2} \subset T \backslash T_{1}$ of cardinality $t_{2}$ is $\left(\begin{array}{c}s \\ s_{2}\end{array}\right)\left(\begin{array}{c}t-t_{1} \\ t_{2}\end{array}\right)$, at most. (We neglect the constraint that $S_{2}$ needs to be a subset of the set of $S$-partners of $T_{1}$-vertices.) The total count of possibilities is bounded by the product of those two.

Lemma 2.4. In the notations of Lemma 2.3,

(i)

$$
N(\mathbf{s}, \mathbf{t}, \boldsymbol{\xi}) \leq t_{1} !\left(\begin{array}{c}
s \\
t_{1}
\end{array}\right)\left(\begin{array}{c}
t \\
t_{1}
\end{array}\right)\left(\begin{array}{c}
s \\
s_{2}
\end{array}\right)\left(\begin{array}{c}
t-t_{1} \\
t_{2}
\end{array}\right) \mathcal{N}_{1}(\mathbf{s}, \mathbf{t}, \boldsymbol{\xi})
$$

(ii) if $\sigma$ is small enough, and

$$
\left(1+\sigma^{1 / 2}\right) s \leq t \leq 2\left(1-\sigma^{1 / 2}\right) s
$$

then

$$
N(\mathbf{s}, \mathbf{t}, \boldsymbol{\xi}) \leq t_{1} !\left(\begin{array}{c}
s \\
t_{1}
\end{array}\right)\left(\begin{array}{c}
t \\
t_{1}
\end{array}\right)\left(\begin{array}{c}
s \\
s_{2}
\end{array}\right)\left(\begin{array}{c}
t-t_{1} \\
t_{2}
\end{array}\right) \mathcal{N}_{2}(\mathbf{s}, \mathbf{t}, \boldsymbol{\xi}) .
$$

Motivated by Lemma 2.1 and Lemma 2.2, in the next section we will focus on subgraphs of $G^{(3)}(m, n)$ of size not exceeding $n^{1-o(1)}$, showing as promised above that the likely edge density of such subgraphs is asymptotic to 1 . It will remain to prove in the last section that whp there are no Pósa sets $S, T$ of low edge density, with $s+t=O\left(n^{1-o(1)}\right)$. Lemma 2.4 will be a key ingredient of that argument.

\section{Edge density of subgraphs of $G^{(3)}(n, m)$}

Let $m=\Theta(n)$ and let $c:=2 m / n$ satisfy $c \geq 3 / 2+\kappa, \kappa>0$ being fixed and arbitrarily small. For such a $c$, an equation

$$
\frac{x f_{2}(x)}{f_{3}(x)}=c, \quad\left(f_{k}(x):=\sum_{j \geq k} \frac{x^{j}}{j !}\right)
$$

has a unique positive root $\lambda$, bounded away from both 0 and $\infty$ for all $n$. 
Lemma 3.1. Assume that $3 / 2<m / n=O(1)$.

(i) For $\varepsilon_{0}=(1 / 3) \ln ^{-1}\left[27 f_{3}(8 \lambda) / 2^{10} \lambda f_{2}(\lambda)\right]$,

$$
P\left(\exists A \subset[n],|A| \leq \varepsilon_{0} \ln n: e(A)>|A|\right) \rightarrow 0 .
$$

Consequently, whp there does not exist an endpoint set $S$ of size below $\varepsilon_{0} \ln n$.

(ii) Let $\sigma_{n} \rightarrow 0$, but $\left(\sigma_{n} \ln n\right) / \ln \ln n \rightarrow \infty$, and let $\rho_{n}=\left(\sigma_{n} \ln n\right)^{-1 / 2}$, so that $\rho_{n} \rightarrow 0$. Then

$$
P\left(\exists A \subset[n], \varepsilon_{0} \ln n \leq|A| \leq n^{1-\rho_{n}}: e(A) \geq\left(1+\sigma_{n}\right)|A|\right) \rightarrow 0 .
$$

In words, with high probability, the edge density of subgraphs induced by sets $A$, of size from $\varepsilon_{0} \ln n$ to $n^{1-o(1)}$, is $1+o(1)$ at most.

Proof of Lemma 3.1. Our random graph $G^{(3)}(n, m)$ is distributed uniformly on the set of all $C(n, m)$ graphs of minimum degree at least 3 , with $m$ edges and $n$ vertices. From a more general result in Pittel and Wormald [22], for $c>3 / 2$ we have: for $n \rightarrow \infty$,

$$
C(n, m) \sim(2 \pi n \operatorname{Var}[Z])^{-1 / 2}(2 m-1) ! ! \frac{f_{3}(\lambda)^{n}}{\lambda^{2 m}} \exp \left(-\eta-\eta^{2} / 2\right)
$$

here $\lambda$ is the root of (3.1), and $Z$ is Poisson $(\lambda)$ conditioned on being at least 3. Probabilistically, (3.1) says that $\mathrm{E}[Z]=2 m / n$. Also $\eta:=c^{-1} \mathrm{E}\left[\left(\begin{array}{l}Z \\ 2\end{array}\right)\right]$. Constant factors aside, the claim is that

$$
C(n, m)=\Theta\left(n^{-1 / 2}(2 m-1) ! ! \frac{f_{3}(\lambda)^{n}}{\lambda^{2 m}}\right) .
$$

Let $d_{1}, \ldots, d_{n} \geq 3$, meeting $\sum_{i} d_{i}=2 m$, be such that there exists a graph with the degree sequence $\mathbf{d}=\left(d_{1}, \ldots, d_{n}\right)$; we call such $\mathbf{d}$ graphical. Existence of graphical d's for large $n, m$ is a weak consequence of (3.5). Let $g(\mathbf{d})$ denote the total number of graphs for a graphical $\mathbf{d}$. Introduce $G_{\mathbf{d}}$, a random graph distributed uniformly on the set of all $g(\mathbf{d})$ graphs of a given graphical d; obviously, $G_{\mathbf{d}}$ equals, in distribution, $G^{(3)} 3(n, m)$, conditioned on $\left\{\mathbf{d}\left(G^{(3)}(n, m)\right)=\mathbf{d}\right\}$. To handle $G_{\mathbf{d}}$ we use a random pairing model, see Bollobás [4], defined as follows.

Introduce a partition of $[2 m]$ into $n$ disjoint subsets $Q_{1}, \ldots, Q_{n},\left|Q_{i}\right|=$ $d_{i}$, and a set $\Omega$ of all $(2 m-1)$ !! pairings $\omega$ of $2 m$ points in $[2 m]$. Each $\omega$ 
induces a unique multigraph: a pair $(u, v) \in \omega$ with $u, v \in Q_{i}$ becomes a loop at vertex $i$; a pair $(u, v) \in \omega$ with $u \in Q_{i}, v \in Q_{j}$ becomes an edge $(i, j)$. Let $\omega$ be random, distributed uniformly on $\Omega$. Let $M G_{\mathbf{d}}=M G_{\mathbf{d}}(\omega)$ denote the random multigraph induced by $\omega$. And let $\Omega_{g}(\mathbf{d})$ be the set of all graphical $\omega$ 's, those for which $M G_{\mathbf{d}}(\omega)$ is a simple graph, i.e. has neither loops nor multiple edges. Then $M G_{\mathbf{d}}(\omega)$, conditioned on $\omega \in \Omega_{g}(\mathbf{d})$, coincides, in distribution, with $G_{\mathbf{d}}$. This implies that, for any graph property $\mathcal{G}$,

$$
\mathrm{P}\left(G_{\mathbf{d}} \in \mathcal{G}\right)=\frac{\mathrm{P}\left(\left\{M G_{\mathbf{d}} \in \mathcal{G}\right\} \cap \Omega_{g}(\mathbf{d})\right)}{\mathrm{P}\left(\Omega_{g}(\mathbf{d})\right)} \leq \frac{\mathrm{P}\left(M G_{\mathbf{d}} \in \mathcal{G}\right)}{\mathrm{P}\left(\Omega_{g}(\mathbf{d})\right)} .
$$

Crucially,

$$
g(\mathbf{d})=\frac{(2 m-1) ! !}{\prod_{i} d_{i} !} \mathrm{P}\left(\Omega_{g}(\mathbf{d})\right)
$$

[4]. We conclude that

$$
\begin{aligned}
\mathrm{P}\left(G^{(3)}(n, m) \in \mathcal{G}\right) & =C(n, m)^{-1} \sum_{\mathbf{d}} \mathrm{P}\left(G_{\mathbf{d}} \in \mathcal{G}\right) g(\mathbf{d}) \\
& \leq \frac{(2 m-1) ! !}{C(n, m)} \sum_{\mathbf{d}} \mathrm{P}\left(M G_{\mathbf{d}} \in \mathcal{G}\right) \prod_{i \in[n]}\left(1 / d_{i} !\right) ;
\end{aligned}
$$

the sums are over all admissible graphical $\mathbf{d}$. So, by (3.5), uniformly for all graph properties $\mathcal{G}$

$$
\mathrm{P}\left(G^{(3)}(n, m) \in \mathcal{G}\right) \leq_{b} n^{1 / 2} \frac{\lambda^{2 m}}{f_{3}(\lambda)^{n}} \sum_{\mathbf{d}} \mathrm{P}\left(M G_{\mathbf{d}} \in \mathcal{G}\right) \prod_{i \in[n]}\left(1 / d_{i} !\right) .
$$

(For brevity, we write $A \leq_{b} B$ when $A=O(B)$ uniformly over parameters involved, and $B$ is too long to compose nicely with the big $O$ notation.) This bound is perfectly tailored for $\mathcal{G}$ 's implicit in (3.2) and (3.3).

Part (i): Denote the probability in (3.2) by $P_{n 1}$. Suppose that for some $\omega$ and $Q=Q(\omega) \subseteq[n],|Q| \leq \varepsilon_{0} \ln n$, the sub(multi)graph of $M G_{\mathbf{d}}(\omega)$ induced by $Q$ has more edges than vertices. Then there exists $k=k(\omega) \in\left[2, \varepsilon_{0} \ln n\right]$ and point sets $Q_{i_{1}}, \ldots, Q_{i_{k}}$ such that the pairing $\omega$ contains $(k+1)$ pairs of points from $Q_{i_{1}} \cup \cdots \cup Q_{i_{k}}$. 
Combining (3.7) and the union bound, we have then

$$
\begin{array}{r}
P_{n 1} \leq_{b} n^{1 / 2} \frac{\lambda^{2 m}}{f_{3}(\lambda)^{n}} \sum_{4 \leq k \leq \varepsilon_{0} \ln n}\left(\begin{array}{l}
n \\
k
\end{array}\right) \frac{(2(k+1)-1) ! !(2(m-k-1)-1) ! !}{(2 m-1) ! !} \\
\quad \times \sum_{\mathbf{d}}\left(\begin{array}{c}
d_{1: k} \\
2(k+1)
\end{array}\right) \prod_{i=1}^{n} \frac{1}{d_{i} !},
\end{array}
$$

$d_{1: k}:=d_{1}+\cdots+d_{k}$.

Using a bound

$$
\sum_{\substack{\delta_{1}+\cdots+\delta_{j}=\delta \\ \delta_{i} \geq 3}} \prod_{i=1}^{j} \frac{1}{\delta_{i} !}=\left[y^{\delta}\right] f_{3}(y)^{j} \leq \frac{f_{3}(y)^{j}}{y^{\delta}}, \quad \forall y>0 .
$$

the second line sum in (3.8) can be bounded

$$
\begin{aligned}
\sum_{3 k \leq d \leq 2 m}\left(\begin{array}{c}
d \\
2(k+1)
\end{array}\right) \sum_{\substack{d_{1}+\cdots+d_{k}=d \\
d_{i} \geq 3}} \prod_{i=1}^{k} \frac{1}{d_{i} !} \sum_{\substack{d_{k+1}+\cdots+d_{n}=2 m-d \\
d_{i} \geq 3}} \prod_{i=k+1}^{n} \frac{1}{d_{i} !} \\
\leq b \sum_{3 k \leq d \leq 2 m}\left(\begin{array}{c}
d \\
2(k+1)
\end{array}\right) \frac{f_{3}(x)^{k}}{x^{d}} \frac{f_{3}(\lambda)^{n-k}}{\lambda^{2 m-d}}
\end{aligned}
$$

for every $x>0$. The ratio of two consecutive terms in the last sum is

$$
\frac{d+1}{d-2 k-1} \frac{\lambda}{x} \leq \frac{3 k+1}{k-1} \frac{\lambda}{x} \leq 7 \frac{\lambda}{x}=\frac{7}{8}<1
$$

if

$$
x=8 \lambda \text {. }
$$

So the sum is of order

$$
\left(\begin{array}{c}
3 k \\
2(k+1)
\end{array}\right) \frac{f_{3}(8 \lambda)^{k}}{(8 \lambda)^{3 k}} \frac{f_{3}(\lambda)^{n-k}}{\lambda^{2 m-3 k}} .
$$

Using this bound, $\left(\begin{array}{l}n \\ k\end{array}\right) \leq n^{k} / k !, 2 m / n=\lambda f_{2}(\lambda) / f_{3}(\lambda)$, and

$$
(2(k+1)-1) ! !=\frac{(2(k+1)) !}{2^{k+1}(k+1) !}
$$


we easily transform (3.8) into

$$
\begin{aligned}
P_{n 1} \leq_{b} m^{-1 / 2} \sum_{k \leq \varepsilon_{0} \ln n} \frac{k(3 k) !}{2^{k}(k !)^{3}} & {\left[\frac{n}{2 m} \frac{\lambda^{3}}{(8 \lambda)^{3}} \frac{f_{3}(8 \lambda)}{f_{3}(\lambda)}\right]^{k} } \\
& \leq{ }_{b} n^{-1 / 2} \sum_{k \leq \varepsilon_{0} \ln n} k\left[\frac{27}{2^{10}} \frac{f_{3}(8 \lambda)}{\lambda f_{2}(\lambda)}\right]^{k} \rightarrow 0,
\end{aligned}
$$

as

$$
\varepsilon_{0}=(1 / 3) \ln ^{-1}\left[\frac{27 f_{3}(8 \lambda)}{2^{10} \lambda f_{2}(\lambda)}\right] .
$$

Part (ii): Let $P_{n 2}$ be the probability in (3.3). This time we need to bound the probability that there exists $A \subset[n]$, of cardinality $k \in\left[\varepsilon_{0} \ln n, n^{1-\rho_{n}}\right]$ that has at least $\ell=\ell(k)=\left\lceil\left(1+\sigma_{n}\right) k\right\rceil$ edges. The counterpart of (3.8) is

$$
\begin{array}{r}
P_{n 2} \leq{ }_{b} n^{1 / 2} \frac{\lambda^{2 m}}{f_{3}(\lambda)^{n}} \sum_{\varepsilon_{0} \ln n \leq k \leq n^{1-\rho_{n}}}\left(\begin{array}{l}
n \\
k
\end{array}\right) \frac{(2 \ell-1) ! !(2(m-\ell)-1) ! !}{(2 m-1) ! !} \\
\times \sum_{\mathbf{d}}\left(\begin{array}{c}
d_{1: k} \\
2 \ell
\end{array}\right) \prod_{i=1}^{n} \frac{1}{d_{i} !}
\end{array}
$$

The bottom sum is bounded by a sum

$$
\sum_{3 k \leq d \leq 2 m}\left(\begin{array}{c}
d \\
2 \ell
\end{array}\right) \frac{f_{3}(x)^{k}}{x^{d}} \frac{f_{3}(\lambda)^{n-k}}{\lambda^{2 m-d}},
$$

with the consecutive terms ratio bounded by $3.01 \lambda / x \leq 0.76$, if $x=4 \lambda$. So, like (3.10),

$$
\begin{aligned}
P_{n 2} \leq_{b} n^{1 / 2} \sum_{\varepsilon_{0} \ln n \leq k \leq n^{1-\rho_{n}}}\left(\begin{array}{l}
n \\
k
\end{array}\right) \frac{(2 \ell-1) ! !(2(m-\ell)-1) ! !}{(2 m-1) ! !} \\
\times\left(\begin{array}{c}
3 k \\
2 \ell
\end{array}\right) \gamma^{k}, \quad \gamma:=4^{-3} \frac{f_{3}(4 \lambda)}{f_{3}(\lambda)} .
\end{aligned}
$$

Here

$$
\left(\begin{array}{c}
3 k \\
2 \ell
\end{array}\right) \leq\left(\begin{array}{c}
3 k \\
2 k
\end{array}\right) \leq_{b}\left(\frac{3^{3}}{2^{2}}\right)^{k}
$$

and

$$
\frac{(2 \ell-1) ! !(2(m-\ell)-1) ! !}{(2 m-1) ! !}=\frac{\left(\begin{array}{c}
m \\
\ell
\end{array}\right)}{\left(\begin{array}{c}
2 m \\
2 \ell
\end{array}\right)} \leq\left(\begin{array}{c}
m \\
\ell
\end{array}\right)^{-1}
$$


Using the last two bounds and

$$
\left(\begin{array}{l}
n \\
k
\end{array}\right) \leq\left(\frac{e n}{k}\right)^{k}, \quad m^{-1 / 2}\left(\frac{m}{\ell}\right)^{\ell} \leq{ }_{b}\left(\begin{array}{c}
m \\
\ell
\end{array}\right)
$$

we simplify (3.12) to

$$
P_{n 2} \leq_{b} n \sum_{\varepsilon_{0} \ln n \leq k \leq n^{1-\rho_{n}}}\left(\frac{n}{k}\right)^{k}\left(\frac{m}{\ell}\right)^{-\ell} \gamma_{1}^{k}, \quad \gamma_{1}:=e 3^{3} 2^{-2} \gamma
$$

Here, since $\ell \geq\left(1+\sigma_{n}\right) k$,

$$
\begin{aligned}
\left(\frac{n}{k}\right)^{k}\left(\frac{m}{\ell}\right)^{-\ell} \gamma_{1}^{k} & \leq\left(\frac{n}{k}\right)^{k}\left(\frac{n}{k\left(1+\sigma_{n}\right)}\right)^{-k\left(1+\sigma_{n}\right)} \gamma_{1}^{k} \\
& \leq\left(\frac{n}{k}\right)^{-k \sigma_{n}} \cdot\left[\left(1+\sigma_{n}\right)^{-\left(1+\sigma_{n}\right)}\right]^{k} \gamma_{1}^{k}
\end{aligned}
$$

The last expression is decreasing for $k \leq n^{1-\rho_{n}}$, because its logarithmic derivative is

$$
\begin{aligned}
&-\sigma_{n} \ln \frac{n}{k}+\sigma_{n}-\left(1+\sigma_{n}\right) \ln \left(1+\sigma_{n}\right)+\ln \gamma_{1} \\
& \leq-\sigma_{n} \ln \frac{n}{k}+\ln \gamma_{1} \leq-\sigma_{n} \rho_{n} \ln n+\ln \gamma_{1} \\
& \quad=-\left(\sigma_{n} \ln n\right)^{1 / 2}+\ln \gamma_{1} \rightarrow-\infty
\end{aligned}
$$

as $\sigma_{n} \ln n \rightarrow \infty$. So

$$
P_{n 2} \leq_{b} \exp \left[-\varepsilon_{0} \sigma_{n}(\ln n)^{2}+O((\ln n) \ln \ln n)\right] \rightarrow 0,
$$

as $\sigma_{n} \ln n \gg \ln \ln n$.

\section{Moderately large, sparse Pósa sets are unlikely}

Let $d_{\max }=d_{\max }(n, m)$ denote the largest vertex degree in $G^{(3)}(n, m)$. Then let $S, T$ be disjoint subsets of $[n]$, of cardinalities $s$ and $t$, with $t<2 s$. In view of Lemma 3.1, part (i), we may and will confine ourselves to $s+t \geq \varepsilon_{0} \ln n$. Lemma 2.4 asserts two upper bounds for the total number of subgraphs $G^{* *}(S \cup T)$ with parameters $\mathbf{s}, \mathbf{t}$ and $\boldsymbol{\xi}$. (See (2.15) for definition of $\xi_{1}$ and $\xi_{2}$.) Let us bound the number of ways to extend this subgraph to a graph on $[n]$, of minimum degree 3 at least, with $m$ edges. 
Recall that the edge set of $G^{* *}(S \cup T)$ does not contain edges between $T$-vertices. So any such extension of $G^{* *}(S \cup T)$ is determined by an induced subgraph $G\left(S^{c}\right)$. Let $d_{i}$ denote the degree of vertex $i \in S^{c}$ in $G\left(S^{c}\right)$. An admissible $\mathbf{d}=\left\{d_{i}\right\}_{i \in S^{c}}$ meets the conditions

$$
d_{i} \geq \begin{cases}3, & i \in S^{c} \backslash T \\ 3-i, & i \in T_{i}, \quad i=1,2,3\end{cases}
$$

and

$$
\sum_{i \in S^{c}} d_{i}=2 m-2 D, \quad D:=\mu+t_{1}=O\left(n^{1-\rho_{n}}\right)=o(n) .
$$

Then, by (2.21) and the definition of $f_{k}(y)$, the number of ways to extend a given $G^{* *}(S \cup T)$ is bounded above by

$$
\begin{aligned}
(2(m-D)-1) ! ! & \sum_{\substack{\mathrm{d} \text { meets } \\
(4.1)-(4.2)}} \prod_{i \in S^{c}} \frac{1}{d_{i} !} \\
= & (2(m-D)-1) ! !\left[y^{2(m-D)}\right] \prod_{i=1}^{3}\left(\sum_{d \geq 3-i} \frac{y^{d}}{d !}\right)^{t_{i}}\left(\sum_{d \geq 3} \frac{y^{d}}{d !}\right)^{n-s-t} \\
& =(2(m-D)-1) ! !\left[y^{2(m-D)}\right] \prod_{i=1}^{3} f_{3-i}(y)^{t_{i}} \cdot f_{3}(y)^{n-s-t} .
\end{aligned}
$$

By the Cauchy integral formula,

$$
\begin{aligned}
{\left[y^{2(m-D)}\right] \prod_{i=1}^{3} f_{3-i}(y)^{t_{i}} } & \cdot f_{3}(y)^{n-s-t} \\
& =\frac{1}{2 \pi i} \oint_{|y|=r} \frac{1}{y^{2(m-D)+1}} \prod_{i=1}^{3} f_{3-i}(y)^{t_{i}} \cdot f_{3}(y)^{n-s-t} d y .
\end{aligned}
$$

Here $n-s-t \sim n$. Using $\left|f_{k}(y)\right| \leq f_{k}(|y|)$, an inequality $([21])$

$$
\left|f_{3}(y)\right| \leq f_{3}(|y|) \exp \left(-\frac{|y|-\operatorname{Re} y}{4}\right)
$$


and selecting $r=\lambda$, we obtain

$$
\begin{aligned}
& \left|\frac{1}{2 \pi i} \oint_{|y|=r} \frac{1}{y^{2(m-D)+1}} \prod_{i=1}^{3} f_{3-i}(y)^{t_{i}} \cdot f_{3}(y)^{n-s-t} d y\right| \\
& \leq_{b} \frac{1}{\lambda^{2(m-D)}} \prod_{i=1}^{3} f_{3-i}(\lambda)^{t_{i}} \cdot f_{3}(\lambda)^{n-s-t} \int_{\theta=-\pi}^{\pi} e^{-(n-o(n)) \lambda(1-\cos \theta) / 4} d \theta \\
& \leq_{b} \frac{1}{n^{1 / 2} \lambda^{2(m-D)}} \prod_{i=1}^{3} f_{3-i}(\lambda)^{t_{i}} \cdot f_{3}(\lambda)^{n-s-t} .
\end{aligned}
$$

And so the number of extensions of a given $G^{* *}(S \cup T)$ is of order

$$
N_{\text {ext }}(\mathbf{s}, \mathbf{t}, \boldsymbol{\xi}):=\frac{(2(m-D)-1) ! !}{n^{1 / 2} \lambda^{2(m-D)}} \prod_{i=1}^{3} f_{3-i}(\lambda)^{t_{i}} \cdot f_{3}(\lambda)^{n-s-t}
$$

at most. Then, multiplying $N_{\text {ext }}(\mathbf{s}, \mathbf{t}, \boldsymbol{\xi})$ by $N_{1}(\mathbf{s}, t, \boldsymbol{\xi})$, the first bound given in Lemma 2.4, we get an upper bound for the total number of graphs on $[n]$ with $m$ edges, such that $S \cup T$ induces a subgraph $G(S \cup T)$ with parameters $\mathbf{s}, \mathbf{t}, \boldsymbol{\mu}$. Multiplying $N(\mathbf{s}, t, \boldsymbol{\xi}) N_{\text {ext }}(\mathbf{s}, \mathbf{t}, \boldsymbol{\xi})$ by $\left(\begin{array}{c}n \\ s, t\end{array}\right) \leq n^{s+t} / s ! t$ !, and dividing by $C(n, m)$, the total number of the $(n, m)$-graphs of minimum degree 3 at least, we obtain a bound $O\left(E_{n, m}(\mathbf{s}, \mathbf{t}, \boldsymbol{\xi})\right)$ for the expected number of Pósa subgraphs with parameters $\mathbf{s}, \mathbf{t}, \boldsymbol{\mu}$, where

$$
\begin{aligned}
& E_{n, m}(\mathbf{s}, \mathbf{t}, \boldsymbol{\xi})=n^{s+t} \frac{\left(2 \mu_{1}+\mu_{2}\right) !}{2^{\mu_{1}} \mu_{1} !} \frac{(2(m-D)-1) ! !}{(2 m-1) ! !} \exp [O(\sigma(s+t))] \\
& \times \frac{\lambda^{2 D}}{2^{s_{2}+t_{2}} f_{3}(\lambda)^{s+t}} \prod_{i=1}^{3} f_{3-i}(\lambda)^{t_{i}} \\
& \times \frac{1}{s ! t !} t_{1} !\left(\begin{array}{c}
s \\
t_{1}
\end{array}\right)\left(\begin{array}{c}
t \\
t_{1}
\end{array}\right)\left(\begin{array}{c}
s \\
s_{2}
\end{array}\right)\left(\begin{array}{c}
t-t_{1} \\
t_{2}
\end{array}\right) .
\end{aligned}
$$

(See (2.14) and (2.28) for $\mu_{1}, \mu_{2}$ expressed through $\xi_{1}$ and $\xi_{2}$. ) In view of (2.6), (2.10), (2.11) and Lemma 3.1, part (ii), if we allow only $s+t \leq n^{1-\rho_{n}}$, $\rho_{n} \rightarrow 0$, which we do, we need to consider only $\mathbf{s}, \mathbf{t}, \boldsymbol{\xi}$ such that

(4.4) $0 \leq s-t_{1} \leq 2 \sigma_{n}(s+t), \quad s_{3}+t_{3} \leq 2 \sigma_{n}(s+t), \quad \xi_{1}+\xi_{2} \leq 2 \sigma_{n}(s+t)$,

where $\sigma_{n} \rightarrow 0$. (See Lemma 3.1 for a more precise definition of $\sigma_{n}, \rho_{n}$.) Our 
remaining task is to show that the sum of $E_{n, m}(\mathbf{s}, \mathbf{t}, \boldsymbol{\xi})$ over the admissible $(\mathbf{s}, \mathbf{t}, \boldsymbol{\xi})$ approaches zero.

To this end, let us first bound $E_{n, m}(\mathbf{s}, \mathbf{t}, \boldsymbol{\xi})$ by a simpler $E_{n, m}^{*}(\mathbf{s}, \mathbf{t}, \boldsymbol{\xi})$ times $\exp (o(s+t))$. First, by (4.2) and (4.4), in the second line of (4.3)

$$
\begin{aligned}
\frac{\lambda^{2 D}}{2^{s_{2}+t_{2}}} f_{0}(\lambda)^{t_{3}}\left[f_{3}(1)\right]^{s_{3}+t_{3}} & =\frac{\lambda^{2(s+t)}}{2^{t}} \exp \left(O\left(s_{3}+t_{3}+s-t_{1}\right)\right) \\
& =\frac{\lambda^{2(s+t)}}{2^{t}} \exp \left(O\left(\sigma_{n}(s+t)\right) .\right.
\end{aligned}
$$

Next, using

$$
(2 a-1) ! !=\frac{(2 a) !}{2^{a} a !}=\Theta\left[\left(\frac{2 a}{e}\right)^{a}\right],
$$

we obtain that the second fraction in the first line of (4.3) is of order

$$
\begin{aligned}
\left(\frac{e}{2 m}\right)^{D}(1-D / m)^{m-D} & =(2 m)^{-D} e^{O\left(D^{2} / m\right)} \\
& =(2 m)^{-t_{1}-\mu} \exp \left(O\left(n^{-\rho_{n}}(s+t)\right)\right) \\
& =(2 m)^{-(s+t+\xi / 2)} \exp \left(O\left(n^{-\rho_{n}}(s+t)\right)\right)
\end{aligned}
$$

$\xi:=\xi_{1}+\xi_{2}$. Further, by (2.7), (2.16) and (2.17),

$$
\frac{\left(2 \mu_{1}+\mu_{2}\right) !}{2^{\mu_{1}} \mu_{1} !}=\frac{\left(2 s+\xi_{1}\right) !}{2^{2 s-t}\left[s-t+t_{1}+\left(\xi_{1}-\xi_{2}\right) / 2\right] !} \exp \left(O\left(\sigma_{n}(s+t)\right)\right) .
$$

Given $\xi$, the last fraction attains its maximum at $\xi_{1}=\xi, \xi_{2}=0$, and it is

$$
\begin{aligned}
& \frac{(2 s+\xi) !}{2^{2 s-t}\left(s-t+t_{1}+\xi / 2\right) !} \\
& \quad=\frac{\left(s+t-t_{1}\right) !(\xi / 2) !}{2^{2 s-t}}\left(\begin{array}{c}
2 s+\xi \\
s-t+t_{1}+\xi / 2, \xi / 2, s+t-t_{1}
\end{array}\right) .
\end{aligned}
$$

The reason behind (4.8) is that the multinomial coefficients are amenable to easy but sharp estimates. The factorial $\left(s+t-t_{1}\right)$ ! combined with $\left(t_{1} ! / s ! t !\right)\left(\begin{array}{c}s \\ t_{1}\end{array}\right)\left(\begin{array}{c}t \\ t_{1}\end{array}\right)$ in (4.3) will later produce another friendly trinomial coefficient.

Using an inequality

$$
\left(\begin{array}{c}
a+b+c \\
a, b, c
\end{array}\right) \leq \frac{(a+b+c)^{a+b+c}}{a^{a} b^{b} c^{c}}
$$


the trinomial coefficient in (4.8) is bounded above by $e^{H_{1}(s, \mathbf{t}, \xi)}$, where

$$
\begin{aligned}
H_{1}(\mathbf{s}, \mathbf{t}, \boldsymbol{\xi})=\left(s-t+t_{1}\right. & +\xi / 2) \ln \frac{2 s+\xi}{s-t+t_{1}+\xi / 2} \\
& +\xi / 2 \ln \frac{2 s+\xi}{\xi / 2}+\left(s+t-t_{1}\right) \ln \frac{2 s+\xi}{s+t-t_{1}} .
\end{aligned}
$$

Consider the first summand. Notice that

$$
s-t+t_{1}+\xi / 2=2 s-t-\left(s-t_{1}\right)+\xi / 2 \leq 2 s-t+\xi / 2,
$$

and $2 s-t>0$. Suppose that $2 s-t \geq \sigma_{n}^{1 / 2}(s+t)$. Then, as $s-t_{1}$ and $\xi$ are of order $O\left(\sigma_{n}(s+t)\right)$, the summand is

$$
(2 s-t) \ln \frac{2 s}{2 s-t}+O\left(\sigma_{n}^{1 / 2}(s+t)\right) .
$$

If $2 s-t \leq \sigma_{n}^{1 / 2}(s+t)$, then, as $x \ln (a / x)$ is increasing for $x \leq a / e$, the summand is bounded above crudely by

$$
\begin{aligned}
(2 s-t+\xi / 2) \ln \frac{2 s+\xi}{2 s-t+\xi / 2} & \leq 2 \sigma_{n}^{1 / 2}(s+t) \ln \frac{3 s}{\sigma_{n}^{1 / 2}(s+t)} \\
\leq & (2 s-t) \ln \frac{2 s}{2 s-t}+2 \sigma_{n}^{1 / 2}\left(\ln \left(1 / \sigma_{n}\right)\right)(s+t) .
\end{aligned}
$$

Thus the summand is always

$$
(2 s-t) \ln \frac{2 s}{2 s-t}+2 \sigma_{n}^{1 / 2}\left(\ln \left(1 / \sigma_{n}\right)\right)(s+t),
$$

at most. For the second summand in (4.10),

$$
\xi / 2 \ln \frac{2 s+\xi}{\xi / 2} \leq \sigma_{n}(s+t) \ln \frac{3 s}{\sigma_{n}(s+t)}=O\left(\sigma_{n}\left(\ln 1 / \sigma_{n}\right)(s+t)\right) .
$$

The third summand in (4.10) is

$$
t \ln \frac{2 s}{t}+O\left(\sigma_{n}(s+t)\right)
$$

Thus

$$
H_{1}(\mathbf{s}, \mathbf{t}, \boldsymbol{\xi}) \leq(2 s-t) \ln \frac{2 s}{2 s-t}+t \ln \frac{2 s}{t}+O\left(\sigma_{n}^{1 / 2}\left(\ln \left(1 / \sigma_{n}\right)\right)(s+t)\right)
$$


uniformly for $2 s-t>0$. The equation (4.7) becomes

$$
\begin{aligned}
& \frac{\left(2 \mu_{1}+\mu_{2}\right) !}{2^{\mu_{1}} \mu_{1} !} \leq \frac{\left(s+t-t_{1}\right) !(\xi / 2) !}{2^{2 s-t}} \\
\times & \exp \left[(2 s-t) \ln \frac{2 s}{2 s-t}+t \ln \frac{2 s}{t}\right] \cdot \exp \left[O\left(\sigma_{n}^{1 / 2}\left(\ln \left(1 / \sigma_{n}\right)\right)(s+t)\right] .\right.
\end{aligned}
$$

Collecting (4.5), (4.6) and (4.12), we conclude that

$$
E_{n, m}(\mathbf{s}, \mathbf{t}, \boldsymbol{\xi}) \leq E_{n, m}^{*}(\mathbf{s}, \mathbf{t}, \boldsymbol{\xi}) \exp \left[O\left(\sigma_{n}^{1 / 2}\left(\ln \left(1 / \sigma_{n}\right)\right)(s+t)\right]\right.
$$

where

$$
\begin{aligned}
E_{n, m}^{*}(\mathbf{s}, \mathbf{t}, \boldsymbol{\xi})= & \frac{(\xi / 2) !}{m^{\xi / 2}} \frac{n^{s+t} \lambda^{2(s+t)} f_{2}(\lambda)^{s} f_{1}(\lambda)^{t-t_{1}}}{(2 m)^{s+t} f_{3}(\lambda)^{s+t} 2^{2 s}} \\
& \times \exp \left[(2 s-t) \ln \frac{2 s}{2 s-t}+t \ln \frac{2 s}{t}\right] \\
& \times \frac{\left(s+t-t_{1}\right) !}{s ! t !} t_{1} !\left(\begin{array}{c}
s \\
t_{1}
\end{array}\right)\left(\begin{array}{c}
t \\
t_{1}
\end{array}\right)\left(\begin{array}{c}
s \\
s_{2}
\end{array}\right)\left(\begin{array}{c}
t-t_{1} \\
t_{2}
\end{array}\right) .
\end{aligned}
$$

Here, recalling again (4.4),

$$
s_{3}, t_{3} \leq 2 \sigma_{n}(s+t), \quad \xi \leq 2 \sigma_{n}(s+t)
$$

Subject to this constraint, let us bound $\sum_{s_{2}, t_{2}, \xi} E_{n, m}^{*}(\mathbf{s}, \mathbf{t}, \boldsymbol{\xi})$. First of all,

$$
\begin{aligned}
\sum_{\xi} \frac{(\xi / 2) !}{m^{\xi / 2}} & \leq \sum_{\xi}\left(\frac{e \xi}{2 m}\right)^{\xi / 2} \\
& \leq \sum_{\xi \geq 0}\left(\frac{e \sigma_{n}(s+t)}{m}\right)^{\xi / 2} \rightarrow 1
\end{aligned}
$$


Secondly,

$$
\begin{aligned}
\sum_{\substack{s_{2}+s_{3}=s \\
s_{3} \leq 2 \sigma_{n}(s+t)}}\left(\begin{array}{c}
s \\
s_{2}
\end{array}\right) & =\sum_{s_{3} \leq 2 \sigma_{n}(s+t)}\left(\begin{array}{c}
s \\
s_{3}
\end{array}\right) \\
& \leq b\left(\begin{array}{c}
s \\
2 \sigma_{n}(s+t)
\end{array}\right) \leq\left(\frac{e s}{2 \sigma_{n}(s+t)}\right)^{2 \sigma_{n}(s+t)} \\
& =\exp \left[O\left(\sigma_{n}\left(\ln \left(2 / \sigma_{n}\right)\right)(s+t)\right)\right]
\end{aligned}
$$

Likewise

$$
\sum_{\substack{t_{2}+t_{3}=t-t_{1} \\
t_{3} \leq 2 \sigma_{n}(s+t)}}\left(\begin{array}{c}
t-t_{1} \\
t_{2}
\end{array}\right)=\exp \left[O\left(\sigma_{n}\left(\ln \left(2 / \sigma_{n}\right)\right)(s+t)\right)\right]
$$

Observing also that

$$
\frac{\left(s+t-t_{1}\right) !}{s ! t !} t_{1} !\left(\begin{array}{c}
s \\
t_{1}
\end{array}\right)\left(\begin{array}{c}
t \\
t_{1}
\end{array}\right)=\left(\begin{array}{c}
s+t-t_{1} \\
s-t_{1}, t_{1}, t-t_{1}
\end{array}\right)
$$

we then have

$$
\sum_{s_{2}, t_{2}, \xi} E_{n, m}^{*}(\mathbf{s}, \mathbf{t}, \xi) \leq E_{n, m}\left(s, t, t_{1}\right) \exp \left[O\left(\sigma_{n}\left(\ln \left(2 / \sigma_{n}\right)\right)(s+t)\right)\right]
$$

where

$$
\begin{aligned}
& E_{n, m}\left(s, t, t_{1}\right):=\frac{n^{s+t} \lambda^{2(s+t)} f_{2}(\lambda)^{s} f_{1}(\lambda)^{t-t_{1}}}{(2 m)^{s+t} f_{3}(\lambda)^{s+t} 2^{2 s}} \\
& \times \exp \left[(2 s-t) \ln \frac{2 s}{2 s-t}+t \ln \frac{2 s}{t}\right] \cdot\left(\begin{array}{c}
s+t-t_{1} \\
s-t_{1}, t_{1}, t-t_{1}
\end{array}\right) .
\end{aligned}
$$

The trinomial coefficient in (4.14) is bounded above by

$$
\exp \left[\left(s-t_{1}\right) \ln \frac{s+t-t_{1}}{s-t_{1}}+t_{1} \ln \frac{s+t-t_{1}}{t_{1}}+\left(t-t_{1}\right) \ln \frac{s+t-t_{1}}{t-t_{1}}\right] \text {. }
$$

Recall that

$$
s-2 \sigma_{n}(s+t) \leq t_{1} \leq s \Longrightarrow 0 \leq s-t_{1} \leq 2 \sigma_{n}(s+t) .
$$


Since

$$
\left(s-t_{1}\right) \ln \frac{s+t-t_{1}}{s-t_{1}}=\left(s-t_{1}\right) \ln \frac{t}{s-t_{1}}+O\left(\left(s-t_{1}\right)^{2} / t\right)
$$

and $x \ln (t / x)$ is increasing for $x<t / e$, we obtain

$$
\begin{aligned}
\left(s-t_{1}\right) \ln \frac{s+t-t_{1}}{s-t_{1}} & \leq 2 \sigma_{n}(s+t) \ln \frac{t}{2 \sigma_{n}(s+t)}+O\left(\sigma_{n}^{2}(s+t)\right) \\
& =O\left(\sigma_{n} \ln \left(1 / \sigma_{n}\right)(s+t)\right)
\end{aligned}
$$

where $\sigma_{n} \ln \left(1 / \sigma_{n}\right) \rightarrow 0$, as $\sigma_{n} \rightarrow 0$. Furthermore,

$$
t_{1} \ln \frac{s+t-t_{1}}{t_{1}}=s \ln \frac{t}{s}+O\left(\sigma_{n}(s+t)\right) .
$$

Turn to the last summand in (4.15). By (4.16),

$$
0 \leq t-t_{1} \leq t-s+2 \sigma_{n}(s+t)<t-s+3 \sigma_{n}(s+t)
$$

Further

$$
\begin{aligned}
\left(t-t_{1}\right) \ln \frac{s+t-t_{1}}{t-t_{1}} & =\left(t-t_{1}\right) \ln \frac{t}{t-t_{1}}+O\left(s-t_{1}\right) \\
& =\left(t-t_{1}\right) \ln \frac{t}{t-t_{1}}+O\left(\sigma_{n}(s+t)\right)
\end{aligned}
$$

Suppose that

$$
t-s+3 \sigma_{n}(s+t)<t / e
$$

which is equivalent to

$$
t<\frac{s\left(1-3 \sigma_{n}\right)}{1-e^{-1}+3 \sigma_{n}}
$$

Then, for $t>s$,

$$
\begin{aligned}
\left(t-t_{1}\right) \ln \frac{t}{t-t_{1}} & \leq\left(t-s+3 \sigma_{n}(s+t)\right) \ln \frac{t}{t-s+3 \sigma_{n}(s+t)} \\
& \leq(t-s) \ln \frac{t}{t-s}+O\left[\sigma_{n}(s+t) \ln \frac{t}{\sigma_{n}(s+t)}\right] \\
& =(t-s) \ln \frac{t}{t-s}+O\left(\sigma_{n} \ln \left(1 / \sigma_{n}\right)(s+t)\right)
\end{aligned}
$$


If $t \leq s$, then $(4.19)$

$$
\begin{aligned}
\left(t-t_{1}\right) \ln \frac{t}{t-t_{1}} & \leq\left(t-s+3 \sigma_{n}(s+t)\right) \ln \frac{t}{t-s+3 \sigma_{n}(s+t)} \\
& \leq 3 \sigma_{n}(s+t) \ln \frac{t}{3 \sigma_{n}(s+t)} \\
& =O\left(\sigma_{n} \ln \left(1 / \sigma_{n}\right)(s+t)\right) .
\end{aligned}
$$

Suppose that

$$
t \geq \frac{s\left(1-3 \sigma_{n}\right)}{1-e^{-1}+3 \sigma_{n}} .
$$

Then $t-s=\Theta(s)$, and so

$$
\left(t-t_{1}\right) \ln \frac{t}{t-t_{1}}-(t-s) \ln \frac{t}{t-s}=O\left(s-t_{1}\right)=O\left(\sigma_{n}(s+t)\right) .
$$

Combining (4.14)-(4.22), we obtain

$$
\begin{aligned}
E_{n, m}(s, t) & :=\sum_{t_{1}} E_{n, m}\left(s, t, t_{1}\right) \\
& \leq E_{n, m}^{*}(s, t) \exp \left[O\left(\sigma_{n}\left(\ln 1 / \sigma_{n}\right)(s+t)\right)\right],
\end{aligned}
$$

where

$$
\begin{aligned}
& E_{n, m}^{*}(s, t):=t \frac{n^{s+t} \lambda^{2(s+t)}}{(2 m)^{s+t} f_{3}(\lambda)^{s+t} 2^{2 s}} f_{2}(\lambda)^{s} f_{1}(\lambda)^{t-s} \\
& \times \exp \left[(2 s-t) \ln \frac{2 s}{2 s-t}+t \ln \frac{2 s}{t}+s \ln \frac{t}{s}+(t-s)^{+} \ln \frac{t}{(t-s)^{+}}\right]
\end{aligned}
$$

here $x^{+}:=\max \{0, x\}$, and $0 \ln (t / 0):=0$. The exponent in (4.24) equals

$$
2 s \ln 2+(2 s-t) \ln \frac{s}{2 s-t}+(t-s) \ln \frac{s}{t}+(t-s)^{+} \ln \frac{t}{(t-s)+},
$$

and the term $2 s \ln 2$ cancels with $2^{2 s}$ in the denominator of the first line fraction in (4.24).

The rest is a bit of calculus. Recalling that $2 m / n=\lambda f_{2}(\lambda) / f_{3}(\lambda)$, and setting $t=x s$, we write

$$
E_{n, m}^{*}(s, t)=s x e^{s H_{1}(x)}
$$


where

$$
\begin{aligned}
& H_{1}(x)=(1+x) \ln \lambda-x \ln f_{2}(\lambda)+(x-1) \ln f_{1}(\lambda) \\
& +(2-x) \ln \frac{1}{2-x}+(x-1) \ln \frac{1}{x}+(x-1)^{+} \ln \frac{x}{(x-1)^{+}}
\end{aligned}
$$

Since

$$
H_{1}^{\prime}(x)=\left\{\begin{array}{l}
\ln \left(\frac{\lambda f_{1}(\lambda)}{f_{2}(\lambda)} \frac{2-x}{x}\right)+\frac{1}{x}, \quad x<1, \\
\ln \left(\frac{\lambda f_{1}(\lambda)}{f_{2}(\lambda)} \frac{2-x}{x-1}\right), \quad x \in(1,2),
\end{array}\right.
$$

and $\lambda f_{1}(\lambda) / f_{2}(\lambda)>2$, we see that $H_{1}(x)$ is unimodal on $(0,2)$, and attains its maximum at $x^{*} \in(1,2)$

$$
x^{*}=\frac{1+2 \lambda f_{1}(\lambda) / f_{2}(\lambda)}{1+\lambda f_{1}(\lambda) / f_{2}(\lambda)},
$$

and

$$
H_{1}\left(x^{*}\right)=\ln \left[\frac{\lambda^{2}}{f_{2}(\lambda)}\left(1+\lambda f_{1}(\lambda) / f_{2}(\lambda)\right)\right] .
$$

Maple shows that the function on the RHS of (4.25) increases with $\lambda$ and it is zero at $\lambda^{*}=5.162717 \ldots$ At the first glance it would seem necessary to put a constraint $\lambda>\lambda^{*}$ in order to claim that, for those $\lambda$ 's, whp there are no Pósa sets of cardinality $|S|+|T| \leq n^{1-o(1)}$.

We can do better though! Indeed, by the unimodality of $H_{1}(x)$,

$$
\begin{gathered}
\max \left\{H_{1}(x): x \in\left[0,1+\sigma_{n}^{-1 / 2}\right] \cup\left[2-2 \sigma_{n}^{1 / 2}, 2\right]\right\} \\
=\max \left\{H_{1}\left(1+\sigma_{n}^{-1 / 2}\right), H_{1}\left(2-2 \sigma_{n}^{1 / 2}\right)\right\} \\
=\max \left\{\ln \frac{\lambda^{2}}{f_{2}(\lambda)}, \ln \frac{\lambda^{3} f_{1}(\lambda)}{f_{2}(\lambda)^{2}}\right\}+O\left(\sigma_{n}^{1 / 2} \ln \left(1 / \sigma_{n}\right)\right) \\
=\ln \frac{\lambda^{3} f_{1}(\lambda)}{f_{2}(\lambda)^{2}}+O\left(\sigma_{n}^{1 / 2} \ln \left(1 / \sigma_{n}\right)\right),
\end{gathered}
$$

as $\lambda f_{1}(\lambda) / f_{2}(\lambda)>2$. As for $x=t / s \in\left[1+\sigma_{n}^{1 / 2}, 2-2 \sigma_{n}^{1 / 2}\right]$, we use $(2.42)$ instead of (2.41) and improve the bound (4.24) by the factor

$$
(s+t)^{2} \exp \left[-(2 s-t) \ln \frac{s}{2 s-t}-(t-s) \ln \frac{s}{t-s}\right] .
$$


So we can re-define

$$
E_{n, m}^{*}(s, t)=s^{3} x(1+x)^{2} e^{s H_{2}(x)},
$$

where

$$
H_{2}(x)=(1+x) \ln \lambda-x \ln f_{2}(\lambda)+(x-1) \ln f_{1}(\lambda)
$$

a linear function! Now

$$
\max \left\{H_{2}(x): x \in[1,2]\right\}=H_{2}(2)=\ln \frac{\lambda^{3} f_{1}(\lambda)}{f_{2}(\lambda)^{2}},
$$

and this function decreases with $\lambda$.

Indeed, introducing $F(\lambda)=\lambda / f_{1}(\lambda)$ that decreases from 1 at $0+$ to 0 at $\infty$, we have

$$
\frac{\lambda^{3} f_{1}(\lambda)}{f_{2}(\lambda)^{2}}=\frac{\lambda^{3} f_{1}(\lambda)}{\left(f_{1}(\lambda)-\lambda\right)^{2}}=\lambda^{2} \frac{F(\lambda)}{(1-F(\lambda))^{2}}
$$

So

$$
\begin{aligned}
\frac{d}{d \lambda} \frac{\lambda^{3} f_{1}(\lambda)}{f_{2}(\lambda)^{2}}= & 2 \lambda \frac{F(\lambda)}{(1-F(\lambda))^{2}}+\lambda^{2} \frac{1+F(\lambda)}{(1-F(\lambda))^{3}} F^{\prime}(\lambda) \\
& \left(\operatorname{using} F^{\prime}(\lambda)=\frac{1}{f_{1}(\lambda)}-\frac{\lambda e^{\lambda}}{f_{1}(\lambda)^{2}}=\lambda^{-1}\left(F(\lambda)-e^{\lambda} F(\lambda)^{2}\right)\right) \\
= & \frac{\lambda F(\lambda)}{(1-F(\lambda))^{3}}\left[3-F(\lambda)-e^{\lambda} F(\lambda)(1+F(\lambda))\right] \\
= & \frac{\lambda F(\lambda)}{(1-F(\lambda))^{3}\left(e^{\lambda}-1\right)} D(\lambda)
\end{aligned}
$$

here

$$
D(\lambda)=(3-\lambda) e^{2 \lambda}-\left(6+\lambda^{2}\right) e^{\lambda}+\lambda+3=\sum_{j \geq 4} d_{j} \lambda^{j}
$$

and

$$
d_{j}=3 \cdot 2^{j}-j 2^{j-1}-j(j-1)-6, \quad j \geq 4 .
$$

By induction on $j \geq 4, d_{j}<0$ for all $j \geq 4$. Hence

$$
\frac{d}{d \lambda} \frac{\lambda^{3} f_{1}(\lambda)}{f_{2}(\lambda)^{2}}<0, \quad \forall \lambda>0
$$


Maple shows that $\lambda^{3} f_{1}(\lambda) / f_{2}(\lambda)^{2}$ attains value 1 at

$$
\lambda^{* *}=4.789771 \ldots
$$

The corresponding average vertex degree

$$
c^{* *}=\frac{\lambda^{* *} f_{2}\left(\lambda^{* *}\right)}{f_{3}\left(\lambda^{* *}\right)}=5.323132 \ldots
$$

It follows that for $m \geq 2.662 n$ the expected number of the likely Pósa sets $(S, T)$ of size $|S|+|T| \leq n^{1-o(1)}$ approaches zero as $n, m \rightarrow \infty$.

Remark 4.1. As a final remark, observe that within the constraints on the $G(S \cup T)$, the dominant contribution to the expected total number of sparse Pósa sets $(S, T)$ comes from those with $G(S \cup T)$ very close to either a cycle on $S$ or an alternating cycle on the bipartition $S+\widehat{T}_{1},\left(\left|\widehat{T}_{1}\right|=|S|\right)$, with the $|S|$ pendant $T_{1}$-vertices each attached to its own $S$-vertex in both cases. It is not difficult to get directly the asymptotic expected number of such extreme subgraphs in our random graph, and it turns out to be essentially the same as the current estimate.

What this likely means is that it is fruitless to search for another constraint on $G(S \cup T)$ with a potential to further decrease $\lambda^{* *}$ via a sharper bound for the expected number of Pósa sets $(S, T)$.

\section{Acknowledgment}

We thank the editors and the referees for a very high quality refereeing process that allowed us to improve readability of the paper.

\section{References}

[1] M. Ajtai, J. Komlós, and E. Szemerédi, First occurrence of Hamilton cycles in random graphs, Cycles in graphs (Burnaby, B.C.) (1982) 173178. MR0821516

[2] J. Aronson, A. M. Frieze, and B. Pittel, Maximum matchings in sparse random graphs: Karp-Sipser revisited, Random Structures and Algorithms 12 (1998) 111-178. MR1637403

[3] T. Bohman and A. M. Frieze, Hamilton cycles in 3-out, Random Structures and Algorithms 35 (2009) 393-417. MR2571776 
[4] B. Bollobás, A probabilistic proof of an asymptotic formula for the number of labelled regular graphs, European J. Comb. 1 (1980) 311316. MR0595929

[5] B. Bollobás, The evolution of random graphs, Trans. Amer. Math. Soc. 286 (1984) 257-274. MR0756039

[6] B. Bollobás, Random Graphs, Second Edition. Cambridge University Press (2001). MR1864966

[7] B. Bollobás, Almost all regular graphs are Hamiltonian, European Journal on Combinatorics 4 (1983) 97-106. MR0705962

[8] B. Bollobás, C. Cooper, T. Fenner, and A. M. Frieze, On Hamilton cycles in sparse random graphs with minimum degree at least $k$, Journal of Graph Theory 34 (2000) 42-59. MR1753066

[9] B. Bollobás, T. Fenner, and A. M. Frieze, Hamilton cycles in random graphs with minimal degree at least $k$, in: A Tribute to Paul Erdos, edited by A. Baker, B. Bollobas and A. Hajnal (1990) 59-96. MR1117005

[10] P. Erdős and A. Rényi, On the evolution of random graphs, Publ. Math. Hungar. Acad. Sci. 5 (1960) 17-61. MR0125031

[11] T. Fenner and A. M. Frieze, Hamiltonian cycles in random regular graphs, Journal of Combinatorial Theory B 40 (1984) 103-112. MR0766628

[12] W. Fernandez de la Vega, Long paths in sparse random graphs, Studia Sci. Math. Hungar. 14 (14) (1979) 335-340. MR0685900

[13] A. M. Frieze, Finding hamilton cycles in sparse random graphs, Journal of Combinatorial Theory B 44 (1988) 230-250. MR0930208

[14] A. M. Frieze, On Hamilton cycles in random graphs with minimum degree at least three.

[15] A. M. Frieze and S. Haber, An almost linear time algorithm for finding Hamilton cycles in sparse random graphs with minimum degree at least three.

[16] A. M. Frieze and B. Pittel, Perfect matchings in random graphs with prescribed minimal degree, in: Trends in Mathematics, Birkhauser Verlag, Basel (2004) 95-132. MR2090500 
[17] R. M. Karp and M. Sipser, Maximum matchings in sparse random graphs, in: Proceedings of the 22nd Annual IEEE Symposium on Foundations of Computing (1981) 364-375.

[18] J. Komlós and E. Szemerédi, Hamilton cycles in random graphs, in: Infinite and finite sets (Colloq., Keszthely, 1973; dedicated to P. Erdos on his 60th birthday) (1975) 1003-1010. MR0392674

[19] A. Korshunov, A solution of a problem of P. Erdős and A. Rényi about Hamilton cycles in non-oriented graphs, Metody Diskr. Anal. Teoriy Upr. Syst. Sh. Trudov. 31 (1977) 17-56 (in Russian).

[20] J. Komlós and E. Szemerédi, Limit distribution for the existence of Hamiltonian cycles in a random graph, Discrete Mathematics 43 (1983) 55-63. MR0680304

[21] B. Pittel, On tree census and the giant component in sparse random graphs, Random Structures and Algorithms 1 (1990) 311-342. MR1099795

[22] B. Pittel and N. C. Wormald, Counting connected graphs inside-out, J. Comb. Theory, Ser. B 93 (2005) 122-172. MR2117934

[23] L. Pósa, Hamiltonian circuits in random graphs, Discrete Mathematics 14 (1976) 359-364. MR0389666

[24] R. Robinson and N. Wormald, Almost all regular graphs are Hamiltonian, Random Structures and Algorithms 5m (1994) 363-374. MR1262985

Alan Frieze

Department of Mathematical Sciences

Carnegie Mellon University

PitTsBurgh, PA 15213

USA

E-mail address: alan@random.math.cmu.edu

Boris PitTel

Department of Mathematics

Ohio State University

Columbus, OH 43210

USA

E-mail address: bgp@math.ohio-state.edu

ReCEIVED April 14, 2011 